\title{
Design Considerations for Self-Powered Wearable Wireless Multimodal Vigilant Sensing Systems
}

\author{
A Thesis \\ Presented to \\ the faculty of the School of Engineering and Applied Science \\ University of Virginia
}

in partial fulfillment

of the requirements for the degree

Master of Science

by

Matthew James Ridder

December 2017 


\title{
APPROVAL SHEET
}

\author{
This Thesis \\ is submitted in partial fulfillment of the requirements \\ for the degree of \\ Master of Science
}

Author Signature: Imattrew- ganes thate

This Thesis has been read and approved by the examining committee:

Advisor: John Lach

Committee Member: Benton Calhoun

Committee Member: Joanne Dugan

Committee Member:

Committee Member:

Committee Member:

Accepted for the School of Engineering and Applied Science:

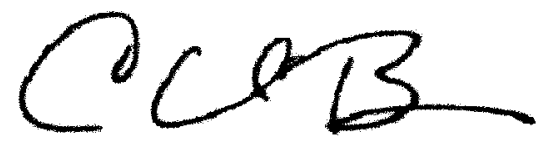

Craig H. Benson, School of Engineering and Applied Science

December 2017 


\section{Abstract}

Body Sensor Networks (BSNs) are cyber-physical sensor systems that address the weaknesses of traditional remote patient monitoring methods in healthcare and provide the opportunity for the continuous collection of high-quality data. However, they must overcome the obstacles of user acceptance and battery life in order for widespread adoption to occur. User acceptance can be addressed by designing BSNs to be wearable and wireless while battery life can be addressed by designing them to have self-powered operation. BSNs must also have multimodal vigilant sensing so that they do not miss any critical events for their given application. One application is cardiac and activity monitoring and it can potentially reduce the number of hospitalizations in patients with a cardiovascular disease (CVD) by tracking CVD symptoms and alerting healthcare professionals when one is detected. One such BSN that performs this kind of monitoring is the testbed for the Self-Powered and Adaptive Low Power Sensing Platform (SAP).

This thesis presents the design of the SAP testbed, which is a self-powered wearable sensor system designed to perform vigilant long-term cardiac and activity monitoring by continuously sensing and wirelessly streaming ECG and motion data to a smartphone. It consumes only $370 \mu \mathrm{W}$ on average and is powered solely by indoor solar allowing it to maintain its monitoring at a vigilant sampling rate so that it does not miss any critical cardiac and activity events. The testbed was deployed on several subjects in the laboratory to validate this behavior. Designing BSNs with similar characteristics to the SAP testbed presents a number of design challenges that must be addressed. These challenges include power management, energy harvesting optimization, system wearability optimization, and system flexibility and modularity optimization. Therefore, in addition to the presentation of the SAP testbed, this thesis discusses the design considerations that must be made in order to make intelligent design decisions when addressing these challenges. 


\section{Acknowledgements}

I would first like to thank my thesis advisor, Professor John Lach, for his valuable guidance during my time at the University of Virginia. His advice and wisdom always helped point me in the right direction at times when I ran into a problem or had a question regarding my research or writing.

I would also like to thank the rest of my committee members, Professors Benton Calhoun and Joanne Dugan, for their time and input in improving the quality of my thesis.

The work presented in this thesis would not have been possible without the support of my colleagues. In particular, I would like to thank Luis Lopez Ruiz, Dawei Fan, Benjamin Ghaemmaghami, Jacob Breiholz, and Jiaqi Gong for their efforts on the SAP testbed over the past couple years.

Finally, I would like to thank my family for supporting me throughout my time at the University of Virginia.

This research was supported in part by the U.S. National Science Foundation under grant number EEC1160483. 


\section{Table of Contents}

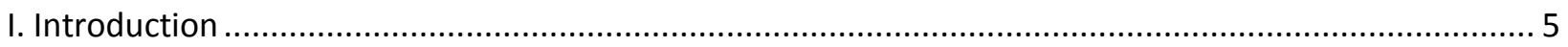

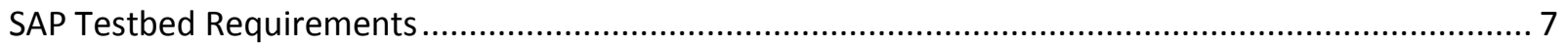

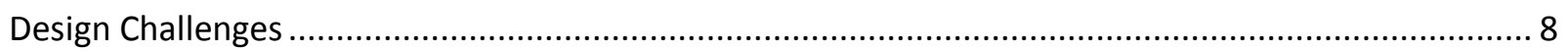

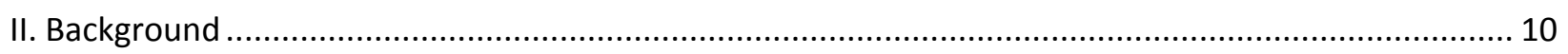

A. Cardiovascular Diseases and Body Sensor Networks ................................................................. 10

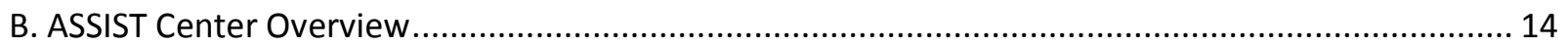

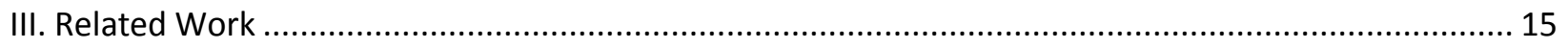

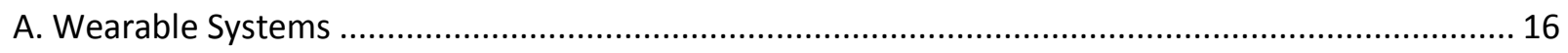

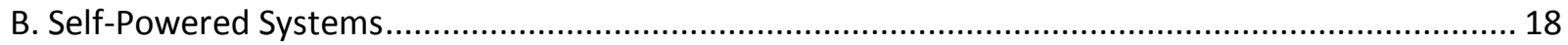

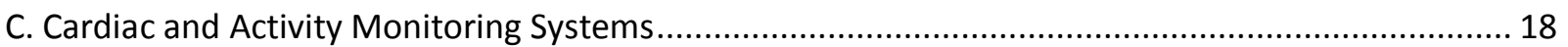

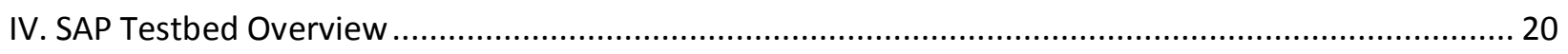

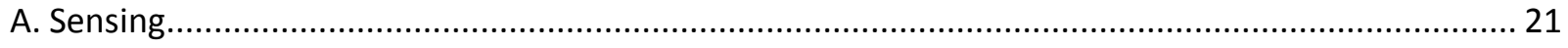

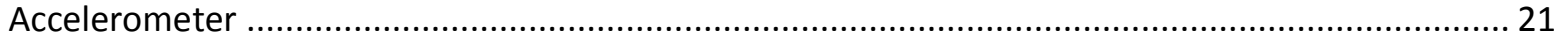

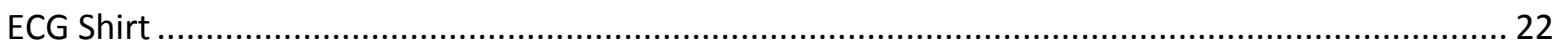

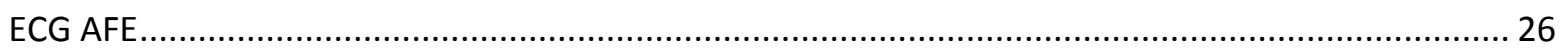

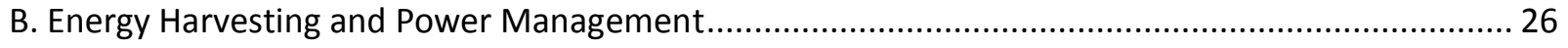

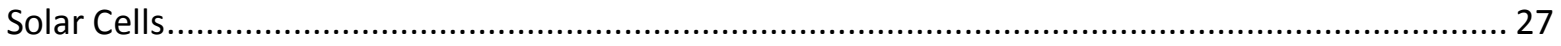

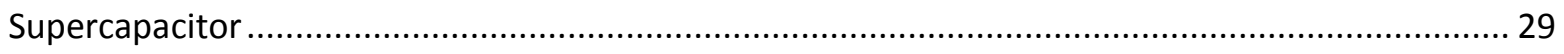

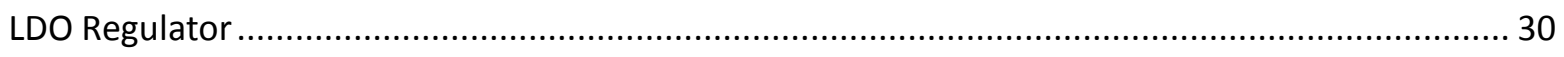

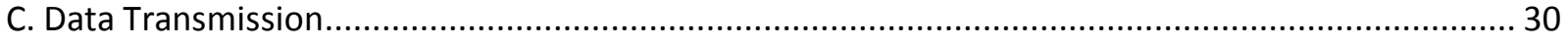

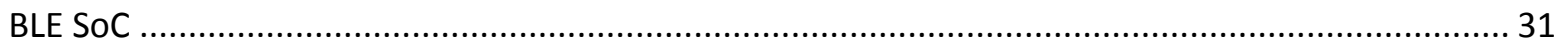

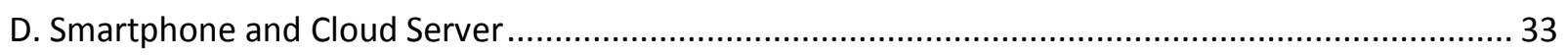

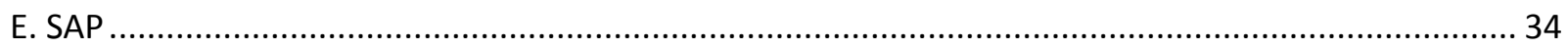

V. Deployment Procedure, Power Consumption, and Results ........................................................... 36

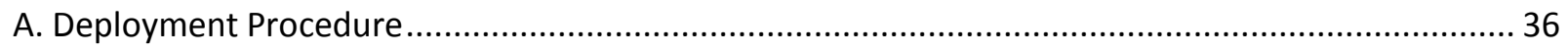

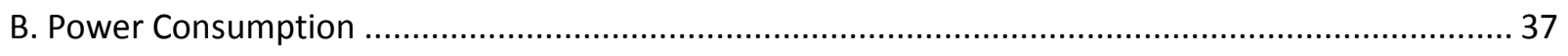

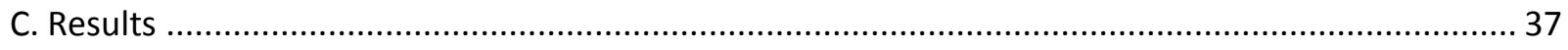

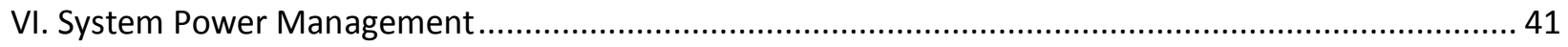

A. Managing Power Consumption in Multimodal Sensing …....................................................... 42

B. Managing Power Generated from a Wearable Energy Harvester ..................................................... 44 


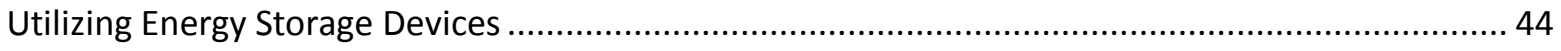

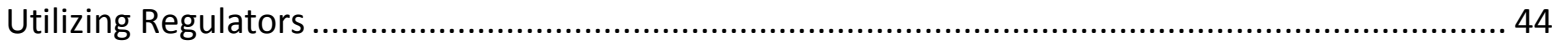

C. Managing Power Consumption in Wireless Communication and Vigilant Sensing .......................... 46

Selecting a Wireless Standard and System on Chip...................................................................... 46

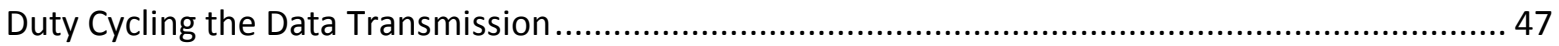

Modeling Power Consumption and Selecting an Optimum Sampling Rate .................................... 48

VII. Energy Harvesting and System Wearability Optimization ............................................................. 52

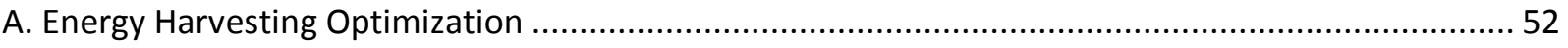

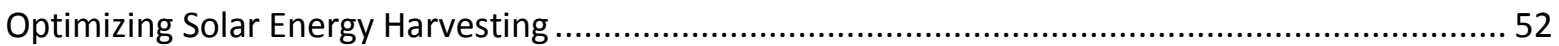

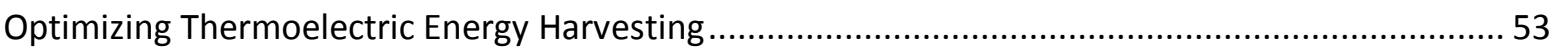

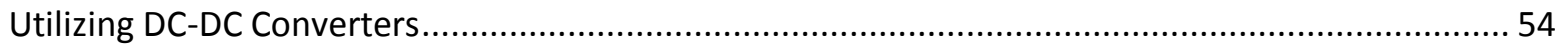

Optimizing Energy Harvesting in the SAP Testbed ....................................................................... 54

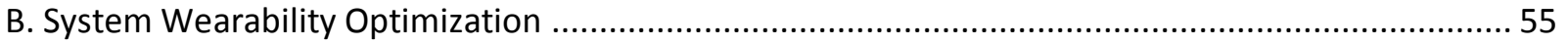

Matching a Sensing Location with an Energy Harvesting Location ............................................. 56

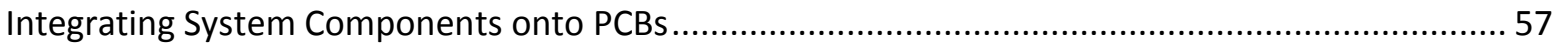

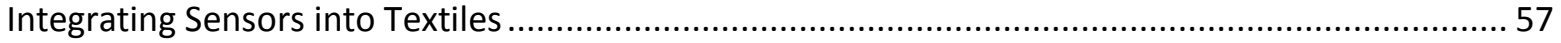

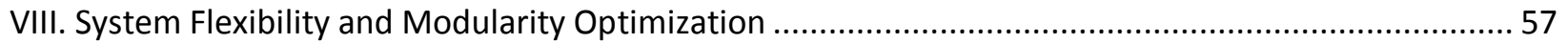

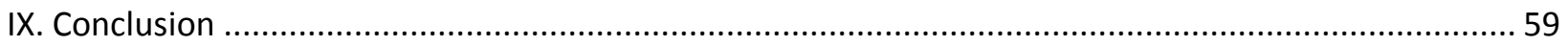

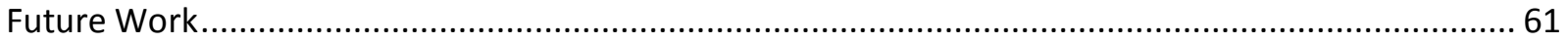




\section{Introduction}

Body Sensor Networks (BSNs) are cyber-physical sensor systems that are capable of collecting, processing, and communicating physiological information from the body and environmental information from the area surrounding it [1]. Although there are many applications for BSNs, much of the BSN research thus far has been done in the context of healthcare and, more specifically, remote patient monitoring. Researchers have used methods of remote patient monitoring such as patient self-report and home health technologies to track symptoms and make recommendations to patients or to intervene when necessary [2]. However, patient self-report tends to be imprecise because it can be difficult for patients to accurately represent their own symptoms, and home health technologies can be a burden to use and only capture infrequent samples of physiological status. BSNs address these weaknesses and have emerged as an option to provide the opportunity for the continuous collection of high-quality data, but they also have formidable obstacles that must be overcome before widespread adoption can occur.

Two of the most significant obstacles that BSNs need to overcome are user acceptance and battery life. These obstacles have prevented widespread adoption because they limit a BSN's ability to provide longterm and continuous monitoring to the user, whether if it is due to the user not willing to wear the BSN or due to the BSN losing power. To address user acceptance, researchers have emphasized the importance of making BSNs wearable and wireless [3]. To address battery life, researchers have emphasized the importance of making BSNs ultra-low power so that they can operate for long periods of time on a small battery or even be powered by an energy harvester attached to the body [4]. If an energy harvester is used and the power that it generates is greater than the power that the system consumes, then the BSN achieves self-powered operation. With self-powered operation, BSNs can operate for longer periods of time than if a battery were used and have the potential to run indefinitely. 
However, achieving such low power to ensure self-powered operation typically requires lower sampling rates and system duty-cycling such that the system is no longer vigilant.

While the term vigilant commonly refers to being alert and watchful, it requires a more specific definition when used in the context of BSNs. In this thesis, vigilant is used to describe a type of sensing for BSNs that is able to detect all specified critical events in its given application. It is important to differentiate vigilant sensing from continuous sensing in that a system with continuous sensing may not collect data at a sampling rate that detects all specified critical events and is therefore not vigilant. Given this refined definition of vigilant, determining what constitutes a critical event is very important and is dependent on the application that the BSN is intended for. For example, if the application of the BSN is to perform ozone monitoring for the purpose of notifying the user when the ozone concentration reaches an unhealthy level, a critical event would be a change in the ozone concentration below a predetermined threshold. If the application is to perform cardiac monitoring for the purpose of notifying the user when a symptom of a cardiovascular disease occurs, a critical event would be an irregular heartbeat. Depending on the application, detecting a critical event can sometimes require multiple sensing modalities which pose additional challenges in BSN design since additional sensors can lead to higher system power consumption.

This thesis discusses the design considerations that must be made in order to make intelligent design decisions when addressing the challenges involved in designing a BSN to be a self-powered wearable wireless multimodal vigilant sensing system. In addition, this thesis presents a BSN with these characteristics that performs cardiac and activity monitoring. This BSN is a testbed that is part of the center for Advanced Self-Powered Systems of Integrated Sensors and Technologies (ASSIST) and is called the Self-Powered and Adaptive Low Power Sensing Platform (SAP). Therefore, the contributions of this thesis are the discussion of the design considerations and the presentation of the SAP testbed. 


\section{SAP Testbed Requirements}

The primary application of the SAP testbed is that it performs vigilant long-term cardiac and activity monitoring for the purpose of detecting critical cardiac and activity events that may indicate the worsening of cardiovascular disease symptoms. Therefore, one requirement of the SAP testbed is that it vigilantly monitors heart activity and motion. The testbed monitors heart activity by continuously measuring an electrocardiogram (ECG) signal with electrodes, and it monitors motion through the use of a 3-axis accelerometer placed on the arm of the user. The critical cardiac event that must be detected in the ECG monitoring is an irregular heart rate, and the critical activity event that must be detected in the motion monitoring is bodily movement. Therefore, the SAP testbed requires a high enough sampling rate to capture every heartbeat as well as small and large movements in order to achieve vigilant cardiac and activity monitoring.

Since the SAP testbed must be integrated onto a person and because its cardiac and activity monitoring must be vigilant and long-term, it has the additional requirements of being wearable, wireless, and having self-powered operation. In order to achieve self-powered operation, the SAP testbed requires an on-body energy harvester that can generate more power than the system consumes. In addition, the testbed must carefully manage its power consumption since its multiple sensors sampled at vigilant sampling rates and its wireless data transmission can consume high amounts of power relative to the power generated by the energy harvester. To ensure that the SAP testbed is wearable, each component of the system needs to be small and compact. Also, the system as a whole needs to be compact and embedded on the body so that it does not burden the user both inside and outside of the laboratory. In order to be wireless, the SAP testbed requires that the ECG and motion data be transmitted using a wireless standard and displayed on a smartphone and cloud server so that the user can view the data locally and the researchers and healthcare professionals can view the data remotely. 
In addition to the application requirements, the SAP testbed must be flexible and modular. Flexibility is required because when the testbed is deployed in the field, there could be a need to add or remove sensing modalities in order to improve the cardiac and activity monitoring or to change the application of the testbed entirely. Modularity is required because ASSIST is a research center and therefore the custom components that the center develops for the SAP testbed are sometimes not readily available. When this occurs, commercial-off-the-shelf (COTS) components have to be used as placeholders. When the ASSIST custom components do become available, the testbed being modular makes the replacement of the COTS components much easier.

\section{Design Challenges}

This thesis presents the design of the SAP testbed for the application of performing vigilant long-term cardiac and activity monitoring. It also discusses the challenges in designing BSNs to be self-powered wearable wireless multimodal vigilant sensing systems, how these challenges are addressed in the SAP testbed, and how the design considerations involved in addressing these challenges can be used to make intelligent decisions when designing other BSNs with similar characteristics. The major design challenges that are addressed in this thesis are:

1. System Power Management (Section VI) - Designing a wearable wireless BSN with multimodal vigilant sensing to have self-powered operation requires effective power management. Therefore, ultra-low power components need to be used in the system, energy storage devices and regulators need to be utilized, the wireless communication needs to be duty cycled, and an optimum sampling rate for the sensors that ensures both vigilant sensing and self-powered operation must be selected.

2. Energy Harvesting Optimization (Section VII.A) - Energy harvesting in a BSN needs to be optimized to ensure that the system can maintain self-powered operation long-term. This can 
be accomplished by choosing a location on the body to place the harvester that maximizes harvesting and by utilizing an efficient DC-DC converter.

3. System Wearability Optimization (Section VII.B) - System wearability needs to be optimized to ensure that the BSN is compact, embedded on the body, and does not affect the daily behavior of the user. This can be done by minimizing the size of the energy harvester, matching the sensing locations to the location of the energy harvester, integrating most of the system components onto small printed circuit boards, and by integrating sensors into textiles.

4. System Flexibility and Modularity Optimization (Section VIII) - Real-world deployments for a BSN can be unpredictable and its functional requirements can change as new information is acquired during research. Therefore, it is important for the BSN to be compatible with different energy harvesters, it must be able to have sensing modalities added or removed, and its architecture must be designed in such a way that enables older technology to be easily replaced with newer technology when it becomes available.

This thesis is organized in the following way. Section II describes the background information related to the application of the SAP testbed, and section III presents the related work. Section IV then gives a detailed overview of the SAP testbed, and section $V$ presents the results from some of the in-lab deployments. Section VI describes the problems and design considerations involved in managing system power consumption, thus addressing challenge 1. Section VII describes the design considerations involved in optimizing energy harvesting and system wearability, thus addressing challenges 2 and 3 . Section VIII then discusses the importance of system flexibility and modularity and the design considerations involved in optimizing them, which addresses challenge 4. Finally, section IX concludes the thesis and presents some ideas for future work. 


\section{Background}

This section describes the background information related to the SAP testbed and its application in order to provide context for the rest of the thesis. This information includes a description of the healthcare problems and costs related to cardiovascular diseases, how BSNs have the potential to improve the healthcare of patients with those diseases, how vigilant cardiac and activity monitoring can be performed by BSNs to detect cardiovascular disease symptoms, and an overview of the ASSIST center.

\section{A. Cardiovascular Diseases and Body Sensor Networks}

Cardiovascular diseases (CVDs) are a leading cause of death in the United States and cost billions of dollars in patient treatment. In fact, in $2010, \$ 324.1$ billion or $14.6 \%$ of the overall cost of healthcare that year was spent on treating people with CVDs [5]. One reason for these high costs is that the worsening of symptoms in CVD patients frequently leads to hospitalization and, even more costly, rehospitalization. This problem of a high number of hospitalizations can be clearly seen in Americans with heart failure. Approximately 50\% of patients with heart failure are re-hospitalized within 6 months of discharge, and $70 \%$ of these re-hospitalizations are related to the worsening of previously diagnosed heart failure [6]. This is also reflected in healthcare costs as a 2003 analysis of Medicare fees for readmission to hospitals showed that heart failure is the number one cause of re-hospitalization [7]. Therefore, being able to reduce the number of re-hospitalizations is of great interest to researchers wanting to reduce the overall cost of healthcare expenditures and to improve the treatment of patients with CVDs.

One way in which researchers have tried to reduce the number of re-hospitalizations is through remote patient monitoring. In remote patient monitoring, health data from patients in one location is transmitted to health care providers in another location. Health care professionals can then assess this 
data and make recommendations to their patients or modify treatment plans accordingly. The potential for remote patient monitoring to reduce the number of re-hospitalizations is best demonstrated in a 2015 study that investigated the results of a remote patient monitoring program for underserved heart failure and chronic obstructive pulmonary disease patients. In the program, researchers measured the rate of emergency department visits and hospital re-admissions for the patients after 30, 90, and 180 days. The study found a 50\% reduction in 30-day re-admissions and a $13-19 \%$ reduction in 180 -day readmission among the patients that received intervention due to the remote patient monitoring [8].

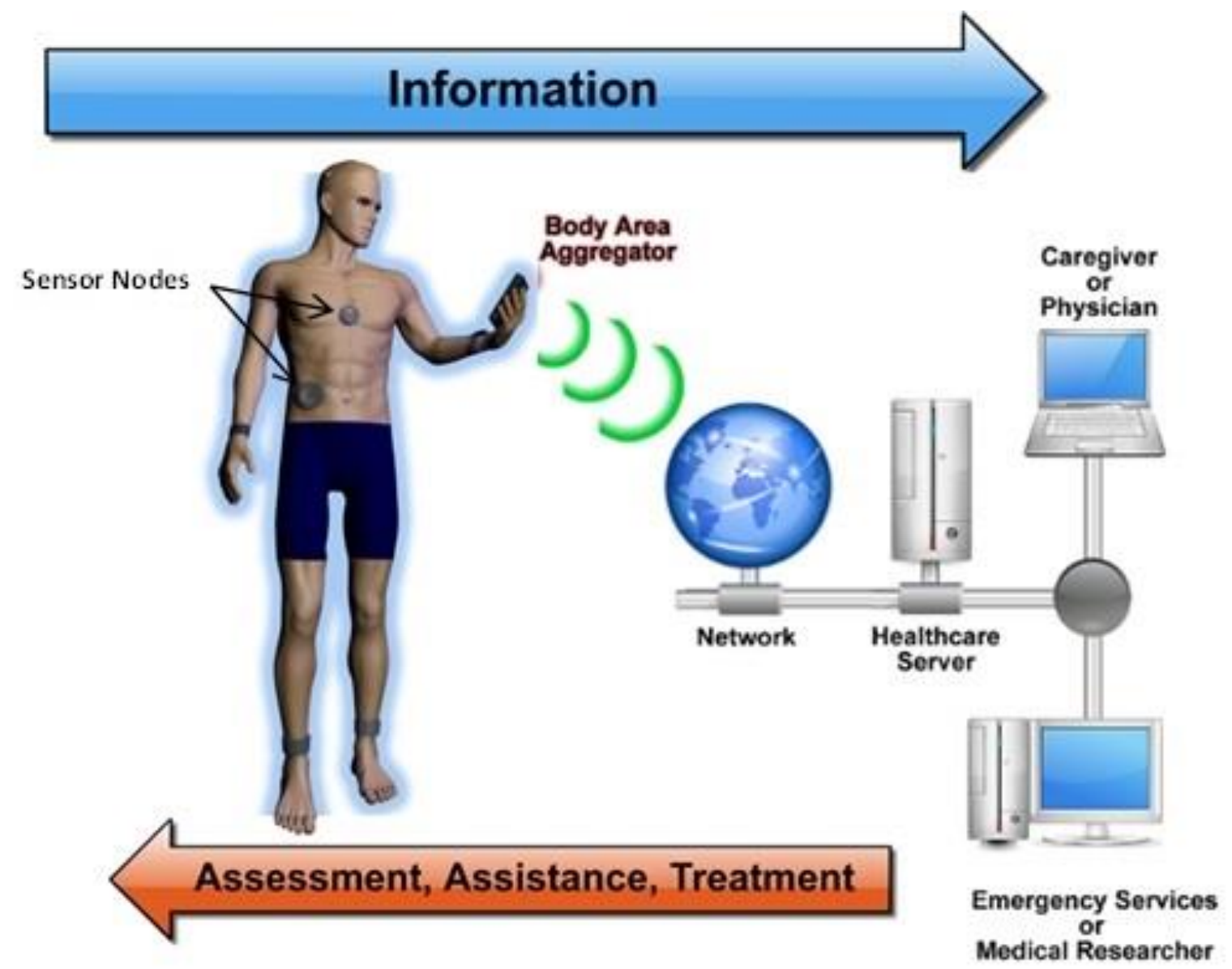

Figure 1. Conceptual representation of a BSN. (Figure is adapted from the INERTIA team website)

Although the findings in this study are promising, there is still a lot of room for improvement in remote patient monitoring. One area of improvement is in how the health data from the patients is collected. In 
the 2015 study, this data was collected by the patients answering a series of questions related to the symptoms of their chronic condition. This method of patient monitoring is commonly referred to as patient self-report. While patient self-report is easy to implement, it has a tendency to be imprecise. Therefore, recording the health data directly from the patients with sensors and wirelessly transmitting it to the healthcare providers may be able to provide more accurate information more frequently, and thus further reduce the number of re-hospitalizations. This can be done through the use of BSNs, and a conceptual representation of one is shown in Figure 1.

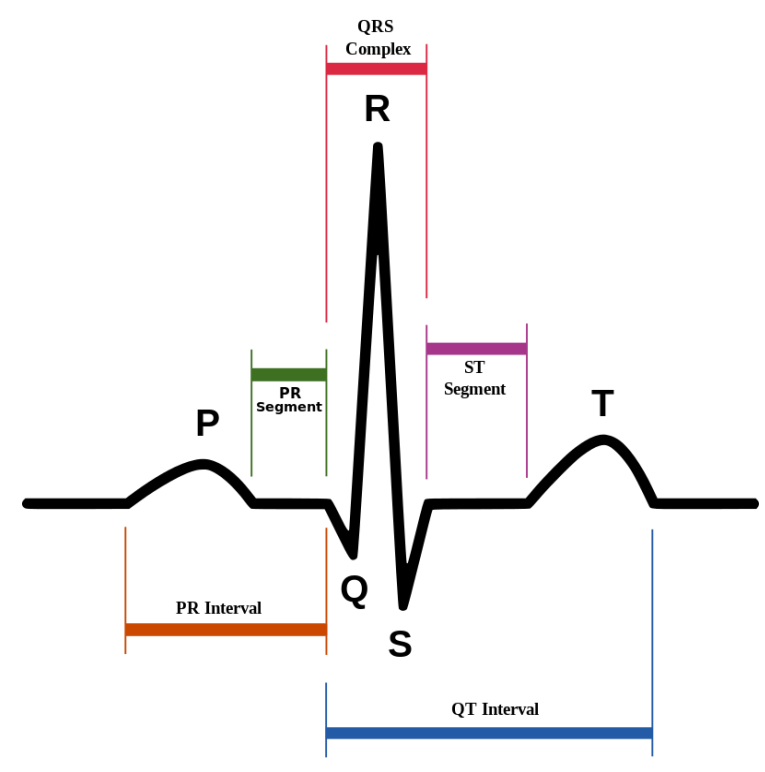

Figure 2. The QRS complex from a typical ECG signal.

For BSNs to record accurate health information from CVD patients, they must perform vigilant cardiac and activity monitoring by detecting all critical cardiac and activity events. Cardiac monitoring is accomplished through electrocardiography in which an ECG signal is measured by placing electrodes on particular points on the chest and back of the user. ECG signals convey a large amount of information about the structure of the heart and how it functions electrically. Some of this information is contained in the periodic waveform known as the QRS complex. This complex is shown in Figure 2 [9]. One valuable piece of information that can be extracted from an ECG signal is heart rate, and it is calculated 
by measuring the time in between the R peaks of consecutive QRS complexes. This time is commonly referred to as the RR interval. Heart rate is particularly useful when monitoring CVD patients because an irregular heart rate (or irregular heartbeat) is a common symptom in many CVDs. Therefore, it can be considered as a critical cardiac event that must be detected. Although other critical cardiac events can be detected by capturing some of the specific features of the QRS complex, they are not considered in this thesis since the SAP testbed only views an irregular heart rate to be a critical cardiac event.

Determining whether or not the patient has an irregular heart rate from an ECG signal alone can often lead to an incorrect diagnosis because ECG signals can easily be distorted by a variety of artifacts [10]. One of the most common of these artifacts is called a motion artifact, and they are particularly common when measuring an ECG signal with a body-worn system. Motion artifacts can move the baseline of the ECG signal and introduce noise that could be interpreted as an additional QRS complex, thus leading to the false detection of an irregular heart rate. Therefore, activity or motion monitoring can be used to determine whether or not there was motion that caused an irregularity in the ECG signal. This monitoring can be accomplished through the use of an accelerometer or gyroscope. In addition to improving the accuracy of the cardiac monitoring, this motion monitoring captures the movement that the user makes in order to record the level of activity of the user. This information can be used by researchers to hypothesize about the relationship between the level of activity and the occurrence of critical cardiac events. Therefore, any bodily movement can be considered as a critical activity event that must be detected.

In addition to performing vigilant cardiac and activity monitoring, BSNs must also be self-powered, wearable, and wireless. However, designing a BSN with these characteristics can be quite challenging with the technology that is commercially available today. Researchers have recognized this challenge 
and have made efforts to develop custom technology that can be used to accomplish this task. One such research center that has been at the forefront of these developments is the ASSIST center.

\section{B. ASSIST Center Overview}

ASSIST is a National Science Foundation sponsored Nanosystems Engineering Research Center that develops and employs nanotechnology enabled energy harvesting and storage, ultra-low power electronics, and sensors to create innovative, battery-free, self-powered, wearable, and wireless health monitoring systems. This system-driven research is accomplished through its five integrated research Thrusts and two Testbeds. Figure 3 shows the technologies that are included in each Thrust and how they and the Testbeds are organized in the ASSIST center [11].

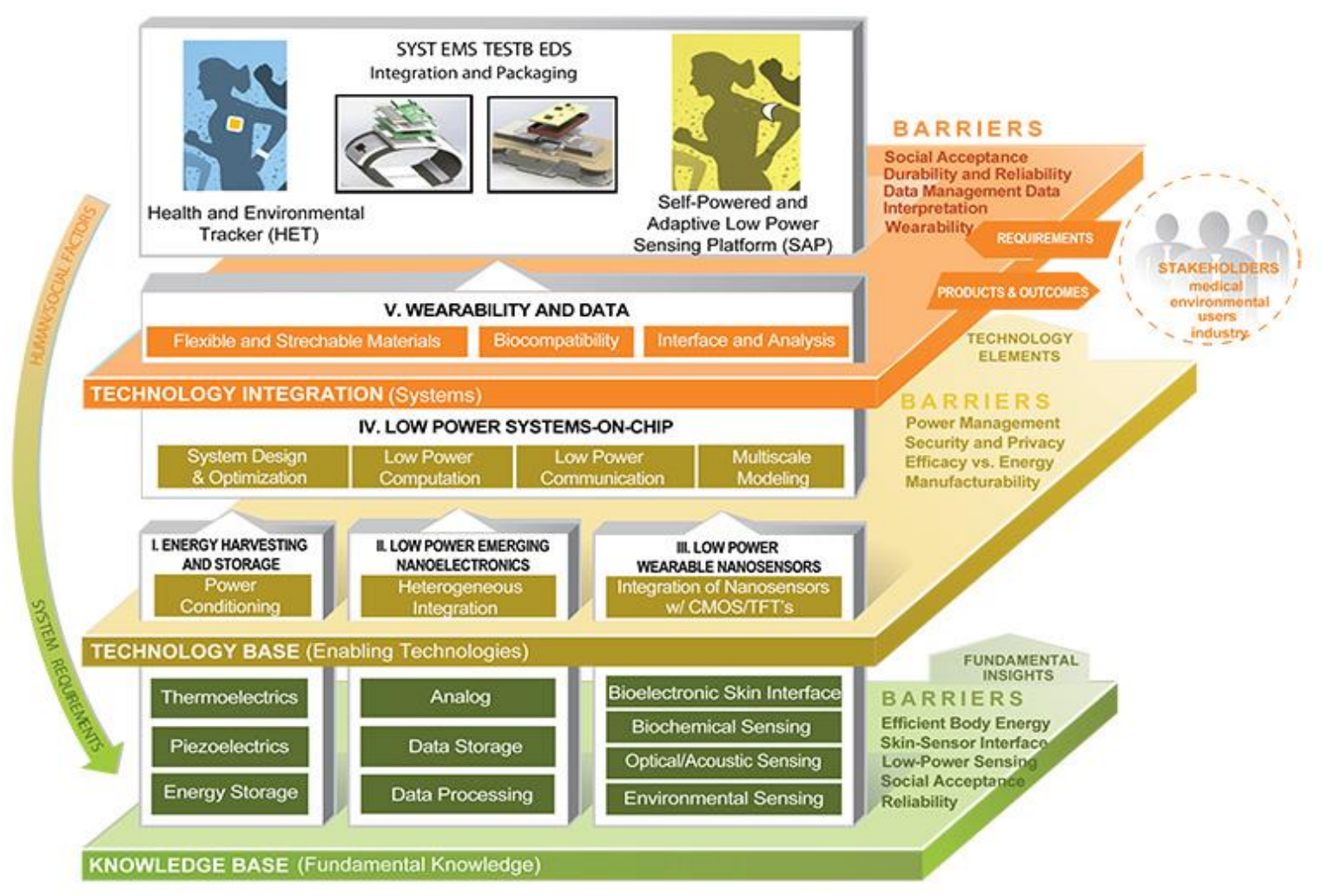

Figure 3. ASSIST Center Thrust and Testbed organization.

This thesis presents the design of the SAP testbed, which is a self-powered wearable wireless BSN that performs vigilant long-term cardiac and activity monitoring by continuously sensing and wirelessly 
streaming ECG and motion data to a smartphone. As a part of the ASSIST Center, this testbed is a collaboration between electrical and materials science engineers from North Carolina State University (NCSU), Pennsylvania State University (PSU), the University of Michigan (UMich), Florida International University (FIU), and the University of Virginia (UVA). The engineers from NCSU and PSU are responsible for developing the technologies in Thrust I, PSU is responsible for Thrust II, FIU is responsible for Thrust III, UVA and UMich are responsible for Thrust IV, and UVA and NCSU are responsible for Thrust V. The engineers from UVA are then responsible for integrating all of the technologies from the different Thrusts into the SAP testbed. Although the purpose of the SAP testbed is to perform vigilant long-term cardiac and activity monitoring in order to mitigate patient symptoms and reduce the number of rehospitalizations in CVD patients, this thesis is strictly focused on the design of the SAP testbed, the challenges that were addressed when designing it, and the design considerations that were made when addressing these challenges and how they can be applied to other self-powered wearable wireless multimodal vigilant sensing systems. Due to the technology readiness level of the testbed, it was only deployed on researchers in the lab and not on actual CVD patients.

\section{Related Work}

Designing a BSN to be self-powered, wearable, and wireless in order to perform vigilant long-term monitoring for a given application is very challenging with the technology that is commercially available today. Due to this challenge, many researchers have not designed their BSNs to have all of these characteristics and have instead focused their efforts on designing them to have just a subset of characteristics to fit the needs of their chosen application. Due to the many potential applications for BSNs in healthcare, there has been an application-based approach to BSN design that has resulted in a wide variety of system designs. The two characteristics in these designs that show the most diversity from application-to-application are system wearability and self-powered operation. This is not surprising 
since the way researchers design their systems to be wearable and/or self-powered is highly dependent on the sensing modalities that the BSN requires and the chosen application. Therefore, the first part of this section describes how researchers have designed BSNs to be wearable, and the second part of this section describes how researchers have designed BSNs to be self-powered. The last part of this section describes the current state of cardiac and activity monitoring systems in order to demonstrate the novelty of the SAP testbed.

\section{A. Wearable Systems}

The authors in [12] present a system that is designed to monitor patients while they perform physical rehabilitation exercises in order to provide them with objective feedback. To accomplish this task, the system uses six modules that embed high-precision accelerometers and wireless communications to transmit the motion data to an acquisition device. Five of these modules act as slaves while one acts as a master. Four of the slaves are used to monitor the motion of the patient's knees and are placed above and below each knee. The fifth slave is placed on the back waist of the patient to provide additional information about the patient's movements. The master module, however, is not attached to the body and is instead connected to a computer through a USB cable and is used to keep the system synchronized while receiving the accelerometer data from the slaves. In order to make the system wearable, the authors designed each module to be as small and compact as possible and used elastic bands to strap each module to their specified locations. However, given the number of the modules and their current sizes, the system is still quite bulky and would likely be a burden for patients that need physical rehabilitation to wear. The authors recognize this and are working to further miniaturize the system in order to improve its wearability.

In [13], the authors present a system that is designed to recognize different forms of human motion such as normal walking, strolling, brisk walking, jogging, and ascending or descending stairs. To achieve 
this goal, they designed a wearable insole based on piezoelectric films. This insole is placed inside of a shoe and uses foot pressure to generate voltage waveforms that contain motion information that can be used to detect the specified motion types. While this system is wearable in the laboratory, it is not wireless and requires the use of an oscilloscope to display the waveforms. Therefore, it would likely be a burden to wear for users outside of the laboratory.

The authors in [14] discuss the design of a system that permits a better understanding of the impact of increased ozone levels and other pollutants on chronic asthma conditions. Their system is comprised of three parts: a wristband, a chest patch, and a handheld spirometer. The wristband sensors track ozone exposure, ambient temperature, ambient relative humidity, photoplethysmography, and motion. The sensors on the chest patch measure ECG, skin impedance, motion, and acoustic signals. Finally, the spirometer measures forced expiratory volume. The system transmits the data from these three devices to a laptop or smartphone using Bluetooth Low Energy. Although this system has many sensing modalities, the authors were able to integrate them all into three small devices which minimize the burden to users both inside and outside of the laboratory.

It is important to note that in this literature review, it is clear that the definition of wearable seems to vary across different groups of authors. For example, the authors in [12] appear to view that a system is wearable if it can be easily attached to the body and does not impede the wearer's movements, while the authors in [13] appear to view that a system is wearable if it is integrated into an article of clothing regardless of whether or not the intended measurements can be easily obtained. The authors in [14], however, appear to have a much more strict definition of wearable and view that a system is wearable if it does not burden the user in any capacity - both in wearing the system and extracting data from it. A more in-depth discussion on system wearability and how the SAP testbed is designed to be wearable is provided in section VII. 


\section{B. Self-Powered Systems}

The authors in [15] present a system that senses body temperature. It consists of a solar cell energy harvester, a power management unit, a microcontroller, a temperature sensor, and a transmitter. All of these are commercial components, and the system consumes $47.2 \mu \mathrm{W}$ with data being transmitted every 134 seconds. The system uses three silicon solar cells in series to harvest energy. Each solar cell has a surface area of $4.865 \mathrm{~cm}^{2}$ and provides $9 \mu \mathrm{W} / \mathrm{cm}^{2}$. The three solar cells in combination generate $132.3 \mu \mathrm{W}$, but due to the power management unit efficiency of $70 \%$, there is only $92.61 \mu \mathrm{W}$ of power available to the system. However, this amount is still sufficient to sustain the system long term, and the authors demonstrate this long-term continuous self-powered operation over indoor lighting conditions ranging from 200 lux to 350 lux. While the system achieves self-powered operation, it is not yet wearable since the system is not in a form factor that can be easily attached to the body. Other authors, however, have designed systems to be both self-powered and wearable.

One example of this is seen in the wearable insole designed by the authors in [13]. As previously discussed, this insole uses piezoelectric films to generate voltage waveforms that can be used to perform motion monitoring. At the same time, these waveforms are used to power the system since they generate an average power of $100 \mu \mathrm{W}$ and the system only consumes $11.9 \mu \mathrm{W}$. This system demonstrates the important concept that there is a relationship between the sensing location and the energy harvesting location in self-powered wearable BSN design. This relationship and how it applies to both the SAP testbed and self-powered wearable wireless multimodal vigilant sensing systems in general is discussed further in section VII.

\section{Cardiac and Activity Monitoring Systems}

Developing cardiac and activity monitoring systems for the purpose of managing cardiovascular diseases is not a novel concept, and there are already commercial systems that perform this task. One example is 
the Alive Bluetooth Heart and Activity Monitor from Alive Technologies [16]. This system monitors ECG and 3-axis motion data in real time and transmits it via Bluetooth to a smartphone. ECG is sampled at $300 \mathrm{~Hz}$ and 3-axis motion is sampled at $75 \mathrm{~Hz}$. As with most commercial systems, the Alive Monitor uses a battery (3.7 V lithium-ion) as its power source and can only last for 48 hours.

The Wearable Context-Aware ECG Monitoring (WCAEM) system [17] is a custom system that performs cardiac and activity monitoring. It contains a wearable ECG sensor with a Bluetooth module that continuously transmits the data to a smartphone. The smartphone displays the data and continuously monitors motion through its built-in kinetic sensors. The ECG sensor in the WCAEM system is sampled from 150 to $300 \mathrm{~Hz}$ and consumes an average of $12.5 \mathrm{~mW}$. Like the Alive Monitor, it has to use a battery for power, in part due to the high sampling rates. These systems are therefore vigilant during their battery lifetime, but are not self-powered.

The Ultra-Low-Power Sensor Evaluation Kit (ULPSEK) [18] is similar to the SAP testbed in that it is selfpowered and performs cardiac and activity monitoring. ULPSEK also measures respiration, body temperature, and photo plethysmography. However, ULPSEK consumes an average of $2.52 \mathrm{~mW}$ when sampling and transmitting its sensor data and must therefore be heavily duty-cycled to preserve selfpowered operation. ULPSEK samples its sensor data for 31 seconds and then sleeps for 12 minutes, reducing the average power consumption over this 12.5 minute window to $137 \mu \mathrm{W}$, which can be supplied by on-body thermoelectric generators. ULPSEK is therefore self-powered but not vigilant, since it can miss critical events when it is asleep.

As described in the remainder of this thesis, the SAP testbed provides vigilant cardiac and activity monitoring with self-powered operation and does so in an integrated, wearable form-factor. The testbed represents a significant advancement in self-powered multi-modal sensing with continuous 
vigilant operation and smartphone/cloud interface capabilities, providing a feasible solution for longterm patient monitoring beyond the clinic.

\section{SAP Testbed Overview}

The SAP testbed is a custom/COTS-based self-powered wearable sensor system designed to perform vigilant long-term cardiac and activity monitoring by continuously sensing and wirelessly streaming ECG and 3-axis motion data to a smartphone. It consumes only $370 \mu \mathrm{W}$ on average and is powered solely by indoor solar allowing it to maintain its ECG and motion monitoring at vigilant sampling rates of $25 \mathrm{~Hz}$ and $8.3 \mathrm{~Hz}$ respectively so that it does not miss any critical cardiac and activity events. The architecture of the SAP testbed is divided into three main blocks: Sensing, Energy Harvesting and Power Management, and Data Transmission. The Sensing block consists of a COTS accelerometer, an ASSIST custom ECG shirt, and an ECG analog front-end (AFE) made from COTS components. The Energy Harvesting and Power Management block consists of COTS solar cells, a COTS DC-DC converter, an ASSIST custom supercapacitor, and a COTS low drop-out (LDO) regulator. Finally, the Data Transmission block consists of a COTS Bluetooth Low Energy (BLE) System on Chip (SoC). A block diagram of this architecture is shown in Figure 4.

Many of the components in the SAP testbed are integrated onto two printed circuit boards (PCBs) in order to make the system more wearable. The two PCBs are the solar boost board and the SAP. The solar boost board contains the DC-DC converter and the supercapacitor needed for integrating the solar cells into the rest of the testbed. The SAP contains the accelerometer, the ECG AFE, the LDO regulator, and the BLE SoC. It acts as the central hub for the testbed to which the ECG shirt and solar boost board connect. In this thesis, SAP only refers to the PCB while SAP testbed refers to the whole system. 


\section{Sensing}

\section{Data Transmission}

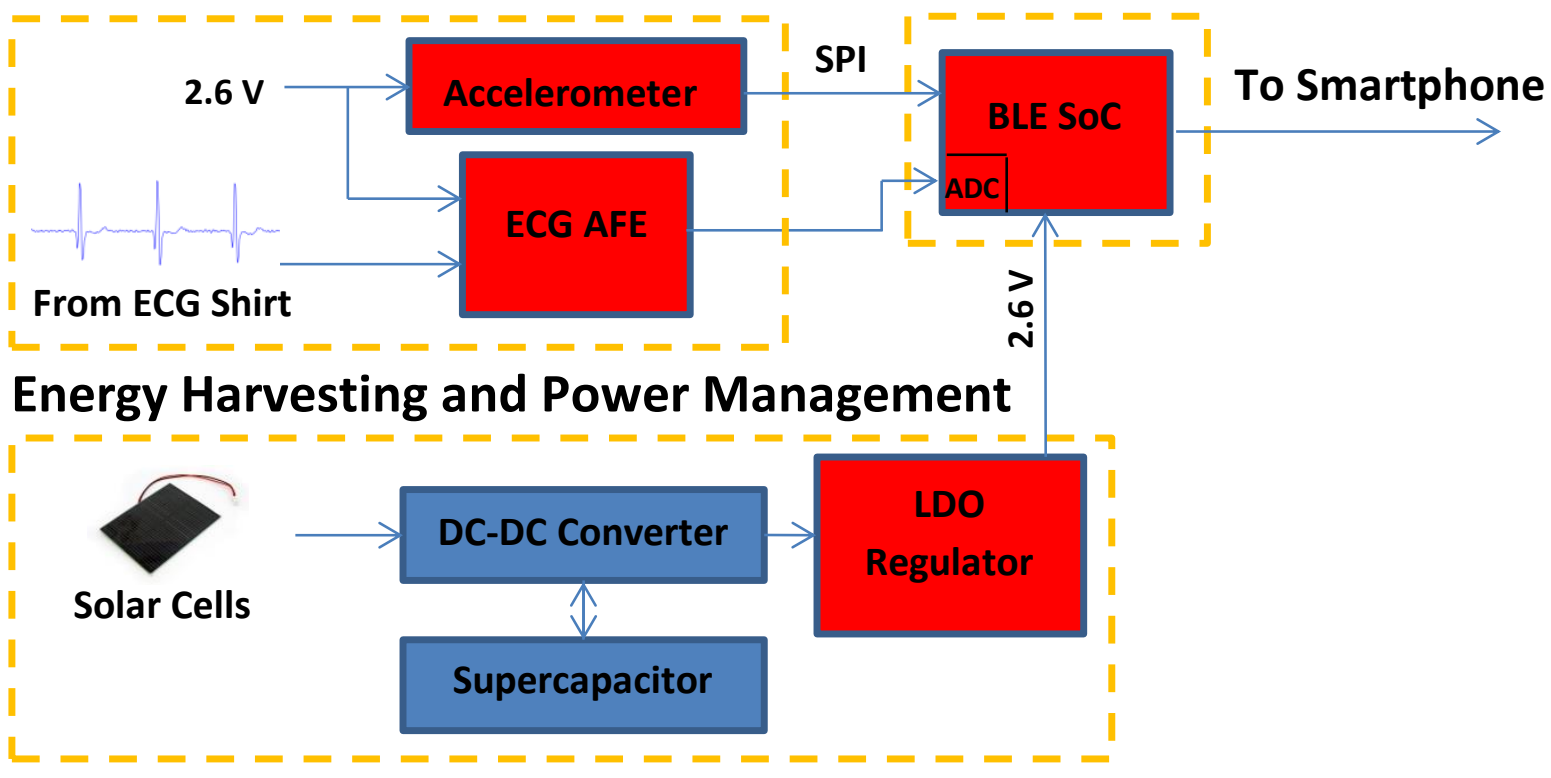

Figure 4. The architecture of the SAP Testbed-blocks in blue are integrated onto the solar boost board while blocks in red are integrated onto the SAP.

\section{A. Sensing}

The function of the Sensing block is to acquire the ECG and motion data from the user and send it to the BLE SoC for transmission. The ECG data is measured with an ECG shirt, and the motion data is measured with a 3-axis accelerometer. The ECG shirt acquires and sends the ECG signal to the BLE SoC through an AFE and an on-chip analog-to-digital converter (ADC). The accelerometer sends the $x_{-}, y_{-}$, and $z$-axis motion data to the BLE SoC through a serial peripheral interface (SPI).

\section{Accelerometer}

The accelerometer used in the SAP testbed is the ADXL362 3-axis digital output MEMS accelerometer from Analog Devices [19]. It uses an I/O voltage range of $1.6 \mathrm{~V}$ to $3.5 \mathrm{~V}$ and operates off of a $1.8 \mathrm{~V}$ to 3.3 $\mathrm{V}$ rail. At $2.6 \mathrm{~V}$ on both the $\mathrm{I} / \mathrm{O}$ and operating rails it consumes less than $5 \mu \mathrm{W}$ of power at a $100 \mathrm{~Hz}$ output data rate making it ideal for ultra-low power use. Additionally, its SPI interface makes it easily 
compatible with the BLE SoC. Figure 5 shows the typical $x-, y^{-}$, and $z$-axis motion data measured from the ADXL362 that is transmitted by the BLE SoC to the smartphone.

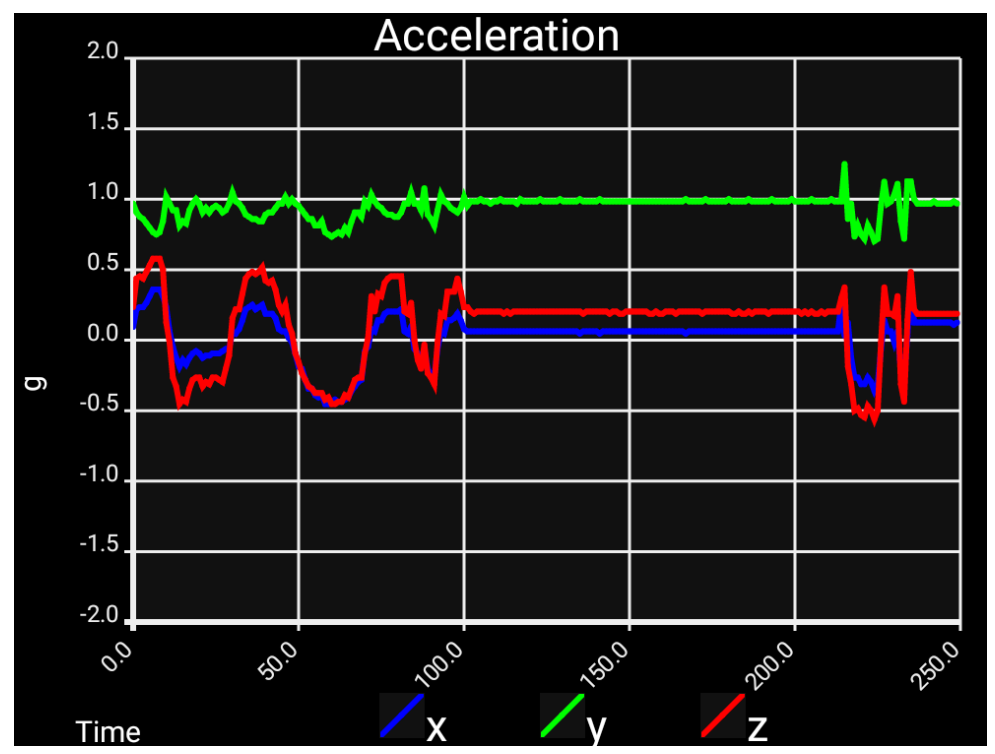

Figure 5. Typical motion data from the accelerometer.

\section{ECG Shirt}

An ASSIST custom ECG shirt designed by engineers at NCSU acquires the ECG signal for the SAP testbed. The shirt has three dry ECG electrodes integrated into a form-fitting fabric similar to the material that Under Armour is made from. In addition to using a form-fitting fabric, the shirt is designed for the dimensions of the intended user so that the shirt conforms to the body as tightly as possible. It is very important that the shirt is form-fitting so that the electrodes have good contact with the body. Without good contact, the electrodes are unable to pick up the ECG signal. Figure 6 shows the ECG shirt. It is shown inside-out in order to display the electrodes. Figure 7 shows the front and back views of the ECG shirt on the body. In both views, it is clear that the shirt has good compression in the areas where the ECG electrodes are located. The three metal knobs in the front view are the snap connection points for the ECG cable that connects the shirt to the SAP. 


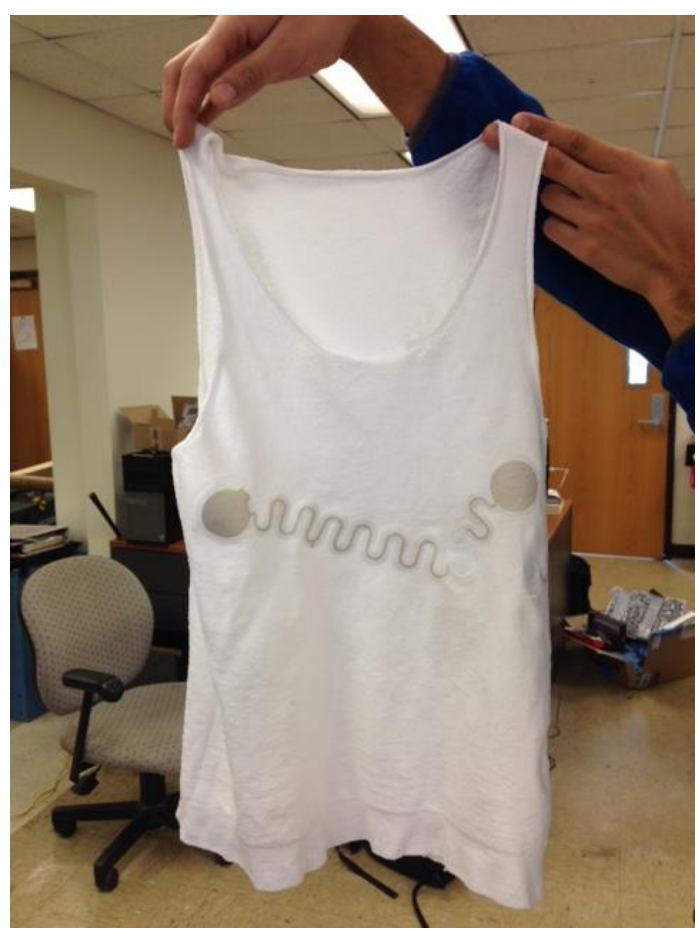

Figure 6. The ECG shirt inside-out.

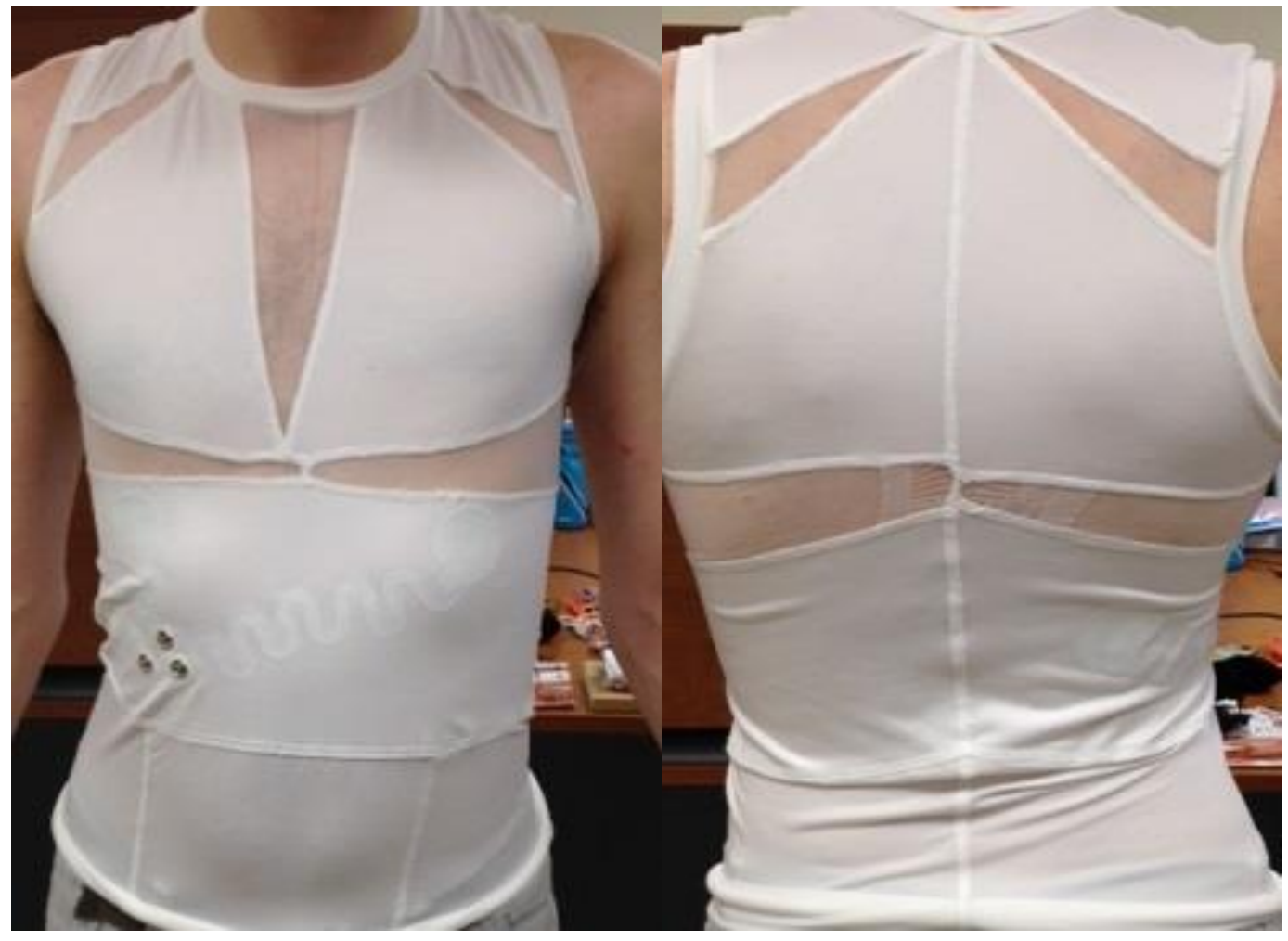

Figure 7. The front and back views of the ECG shirt on the body. 
Although the shirt clearly has good compression in most areas, it can sometimes be difficult to get the electrodes to establish a connection. One reason for this is that taking the shirt off and putting in back on several times can cause the shirt to get wrinkled in a few places. This wrinkling can be seen in the back view of Figure 7. Wrinkling is problematic because it can cause the electrodes to lose contact with the skin. Getting the electrodes to establish a good connection can also be difficult due to dry skin. This is a problem regardless of whether or not each electrode has good contact with the skin because dry skin is a bad conductor and can make it difficult for the electrodes to pick up a signal. Fortunately, there are a couple of solutions to these problems. The first, and probably least practical in day-to-day situations, is to work up a sweat. This moisturizes the skin which makes it a better conductor and helps stick the electrodes in place preventing wrinkles from moving them around. The second solution is to put lotion on the skin where the electrodes make contact. This has a similar effect to working up a sweat but is much more practical.

Once the electrodes establish a connection, the connection and acquired signal are quite robust. This was verified by engineers at UVA by measuring the ECG signal from the shirt when performing different movements and comparing those signals to an ECG signal acquired when performing no movement. These tests were also conducted to ensure that the electrodes maintained contact with the skin throughout the different movements. The motions performed were running in place and dancing. Running in place was performed in order to test the effect of consistent movement while dancing was performed in order to test the effect of erratic movement. The results of these tests are shown in Figures 8,9 , and 10. Figure 8 shows the ECG signal when the user is standing still, Figure 9 shows the ECG signal when the user is running in place, and Figure 10 shows the ECG signal when the user is dancing. Figures 9 and 10 show that both types of movement introduce some noise into the ECG signal, with the noise from running in place being more consistent than the dancing. However, the ECG signal 
was never lost nor was the noise large enough to significantly distort the waveform and prevent the measurement of key features from the QRS complex.

\section{Agilent Technologies}

Wed Apr 05 16:05:052017

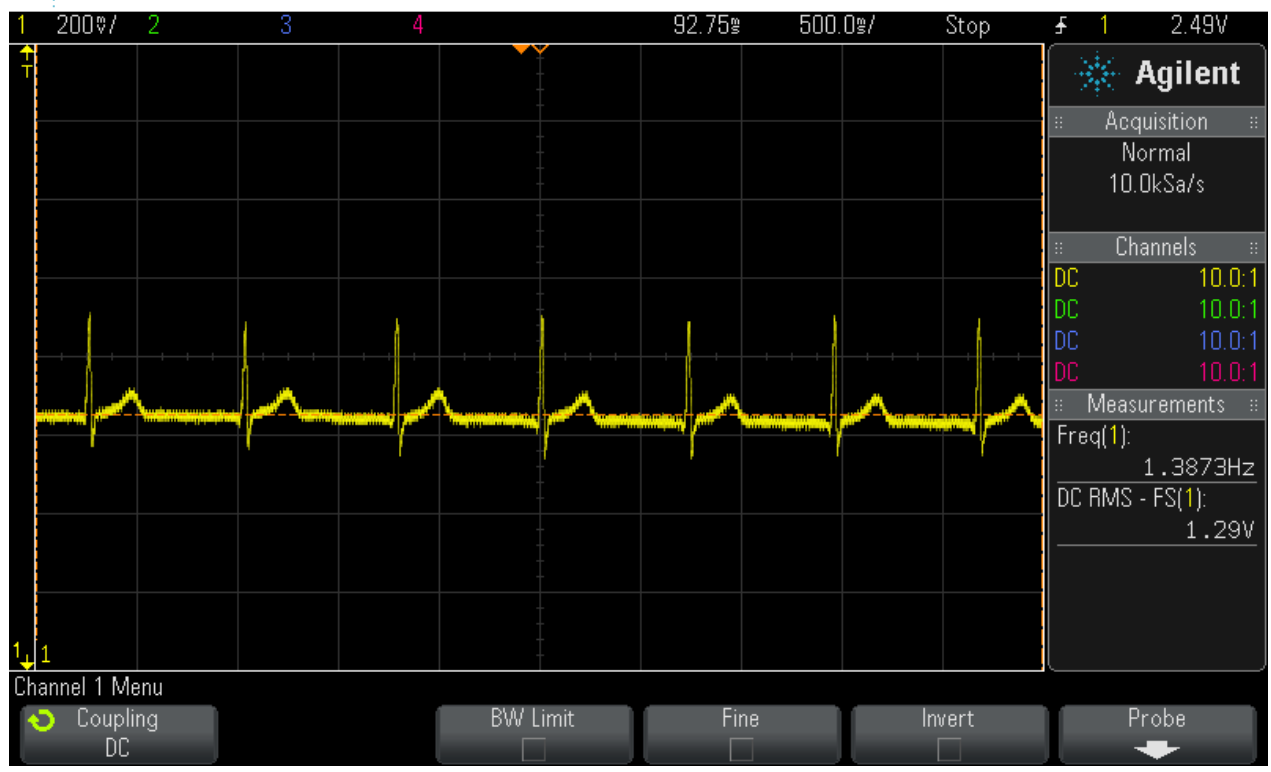

Figure 8. The ECG signal acquired from the shirt: no movement.

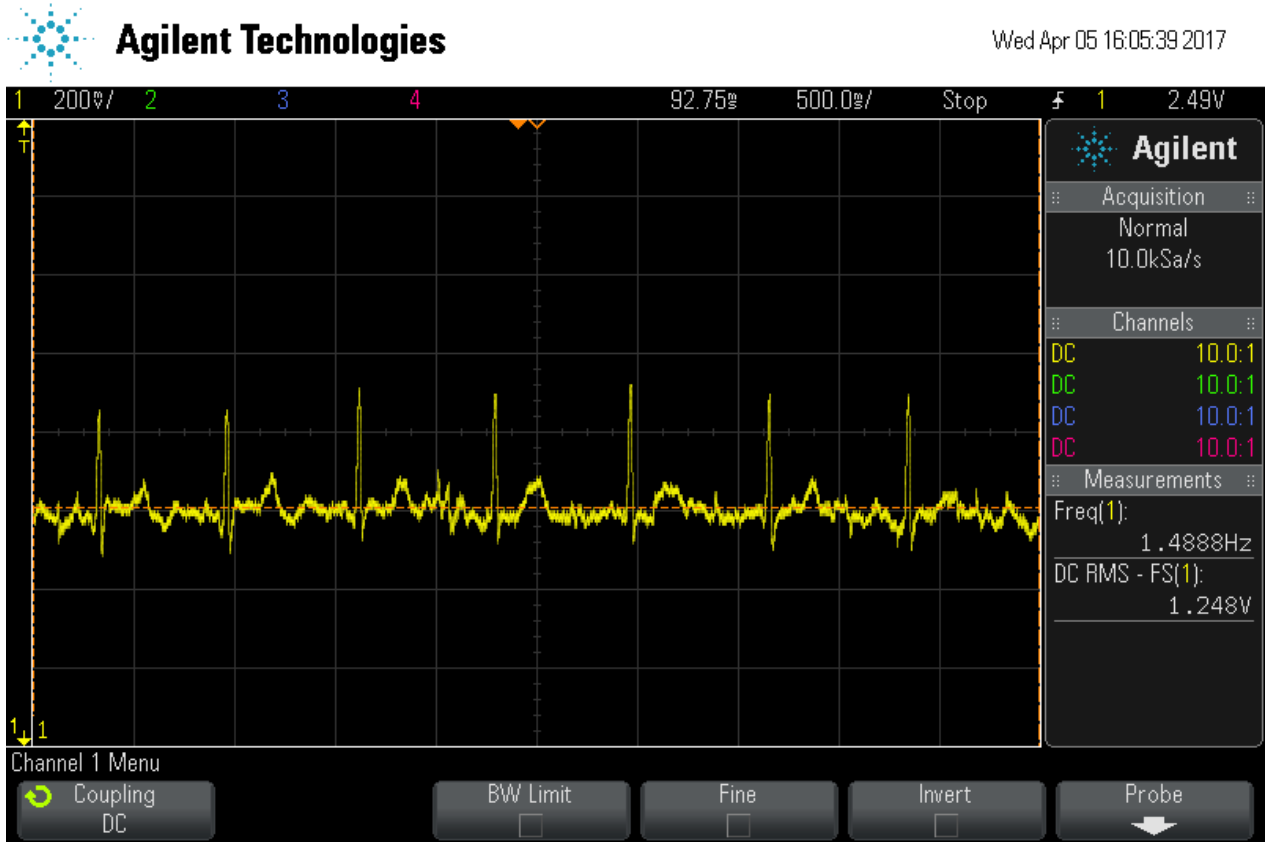

Figure 9. The ECG signal acquired from the shirt: running in place. 


\section{Agilent Technologies}

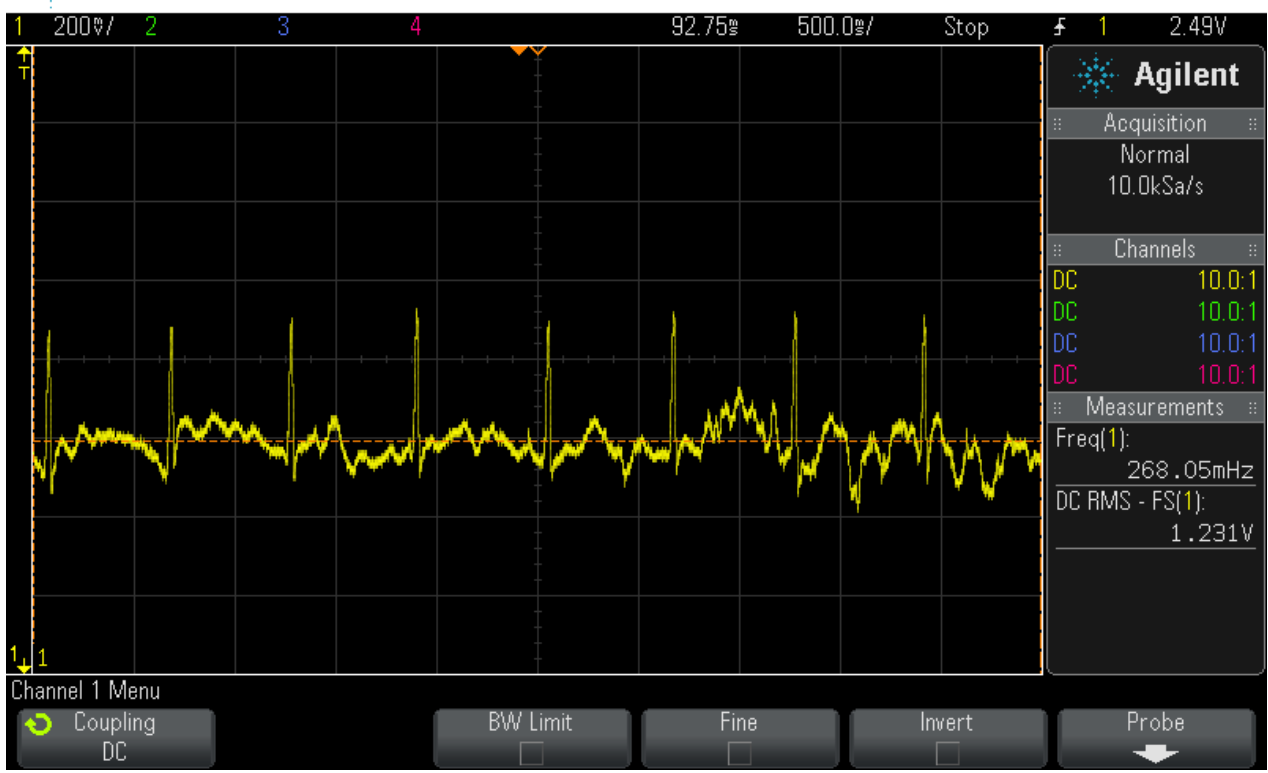

Figure 10. The ECG signal acquired from the shirt: dancing.

\section{ECG AFE}

The ECG AFE consists of five LPV521 operational amplifiers (op-amps) from Texas Instruments [20] and one LT6656 voltage reference from Linear Technology [21]. It is designed to amplify the ECG signal acquired from the ECG shirt 100x so that it can be digitized by the on-chip ADC with high resolution. The LPV521 operates from $1.6 \mathrm{~V}$ to $5.5 \mathrm{~V}$ and has a supply current of about $400 \mathrm{nA}$. The LT6656 generates a $1.25 \mathrm{~V}$ reference that acts as the input to one of the op-amps and has a supply current of $850 \mathrm{nA}$. Both of these low supply currents make these devices ideal for ultra-low power use.

\section{B. Energy Harvesting and Power Management}

The Energy Harvesting and Power Management block administers and supplies power to the Sensing and Data Transmission blocks by extracting available power from the solar cells. The solar cells generate a DC voltage that is sent to a DC-DC converter that supplies the input voltage to the LDO regulator and 
charges the supercapacitor. The LDO regulator then creates the $2.6 \mathrm{~V}$ rail needed for powering the accelerometer, the ECG AFE, and the BLE SoC.

\section{Solar Cells}

The selected solar cells for the SAP testbed are the MP3-37 from Power Film Solar [22]. They are flexible cells that have a nominal open circuit voltage of $3.2 \mathrm{~V}$ and a maximum power output of $3.6 \mathrm{~mW} / \mathrm{cm}^{2}$ under direct sunlight. Two of these cells (with a combined surface area of $83.22 \mathrm{~cm}^{2}$ ) are sewn onto a flexible arm sleeve to optimize the wearability and integration of the system. This arm sleeve is shown in Figure 11. A characterization of the two solar cells on this arm sleeve under common indoor illumination levels [23] is presented in Figure 12, where the maximum power point (MPP) for each condition is indicated by a red star. The output of the solar cells is connected to the BQ25504 Ultra Low-Power Boost Converter from Texas Instruments [24]. This DC-DC converter is responsible for tracking the MPP of the power generated by the solar cells and extracting the maximum power available with high efficiency. This efficiency is typically between $80 \%$ and $90 \%$. The energy extracted is then distributed over two lines: the main one to power the SAP and the secondary one to store the excess energy onto the supercapacitor that powers the SAP when there is not enough energy available at the input to the DCDC converter. External circuity is used to program the DC-DC converter's output voltage to $3 \mathrm{~V}$. The DCDC converter, its external circuitry, and the supercapacitor are integrated onto the solar boost board. This board is shown in Figure 13.

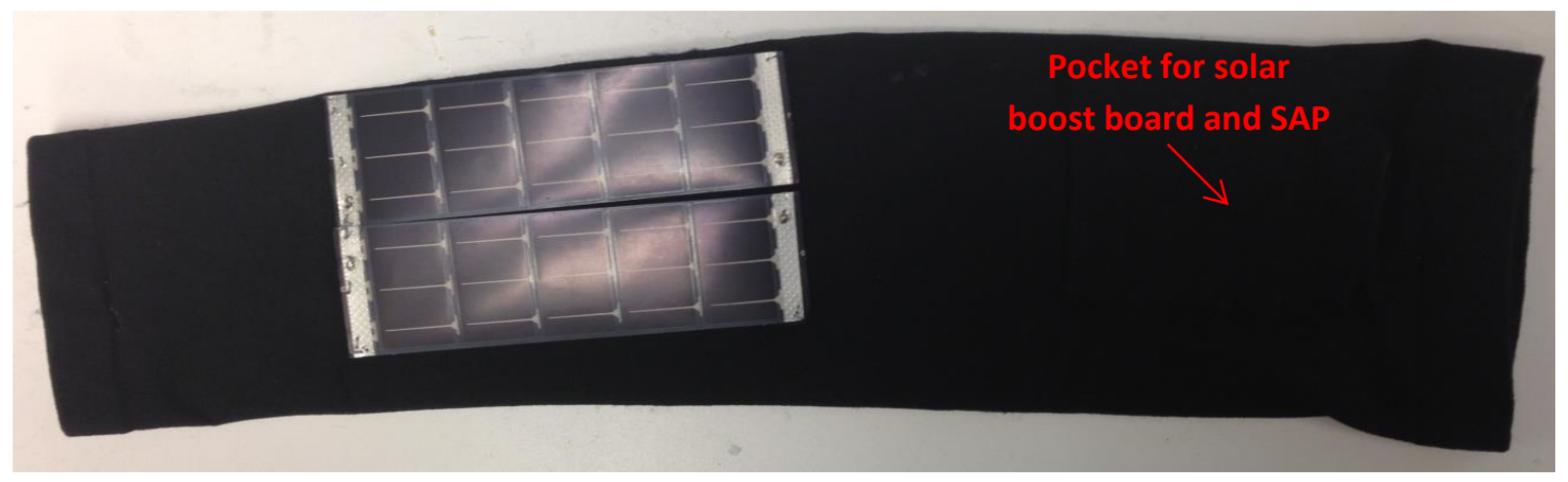

Figure 11. The solar cell arm sleeve. 


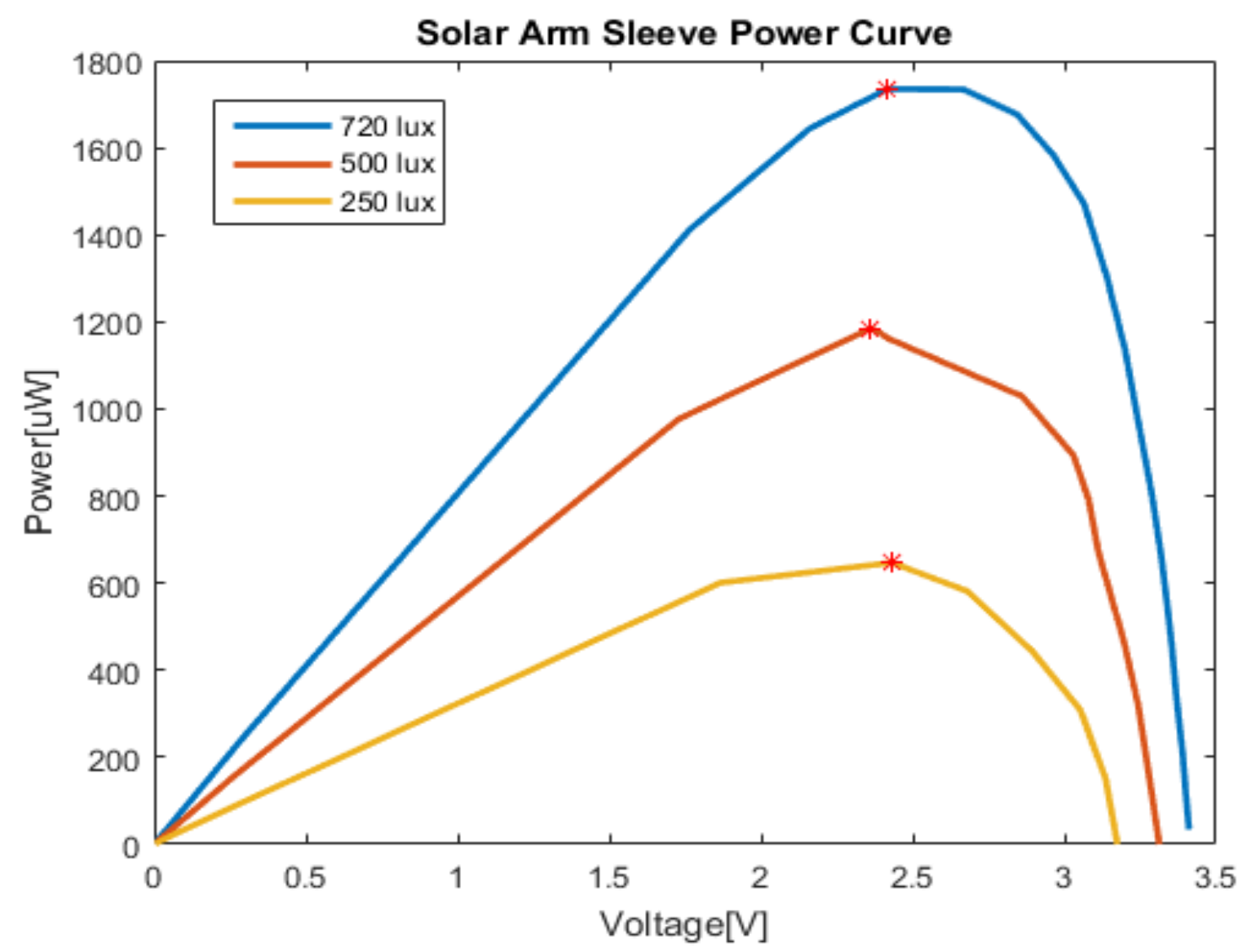

Figure 12. The characterization of the solar arm sleeve for three main levels of illumination in indoor environments. The red start indicates the maximum power point.

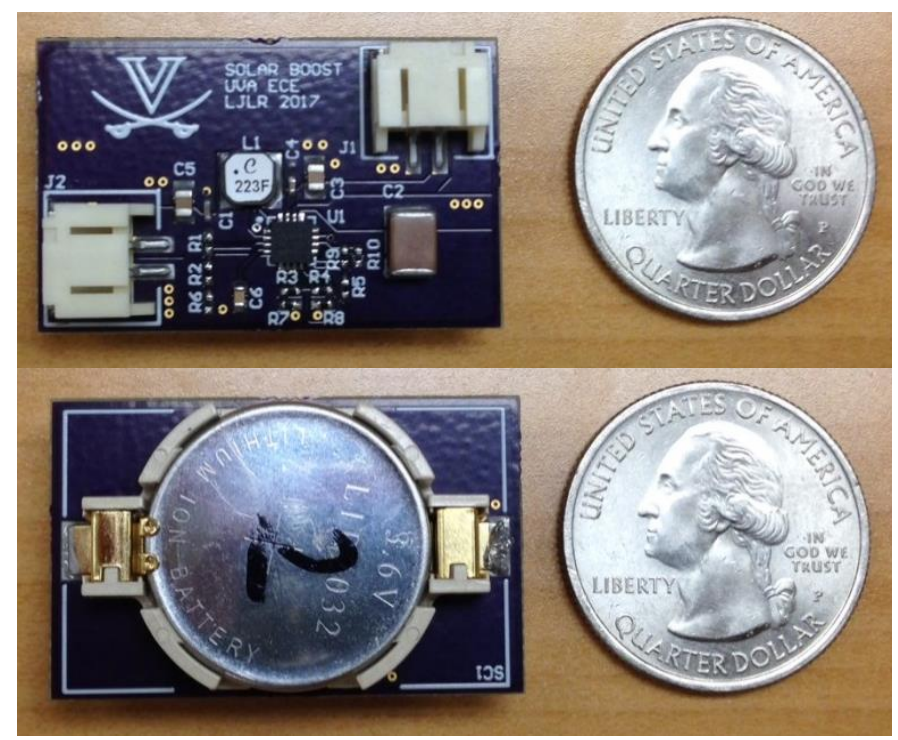

Figure 13. The front (top) and back (bottom) of the solar boost board. 


\section{Supercapacitor}

The SAP testbed uses one of many ASSIST custom supercapacitors for storing harvested energy. These supercapacitors are developed by engineers at PSU and have difference capacitance values ranging from $37 \mathrm{mF}$ to $540 \mathrm{mF}$. Each capacitor is packaged in a small coin cell which enables them to be easily integrated into the solar boost board interchangeably. This coin cell is shown in Figure 14 . Since the packaging for each capacitor is the same, choosing which capacitor to use in the testbed depends on its capacitance and corresponding discharge time. This is discussed further in section VI.B. Table 1 shows the discharge times of the different ASSIST custom supercapacitors, and Figure 15 shows the charge/discharge curve for the $540 \mathrm{mF}$ capacitor as an example of the behavior of these custom supercapacitors.

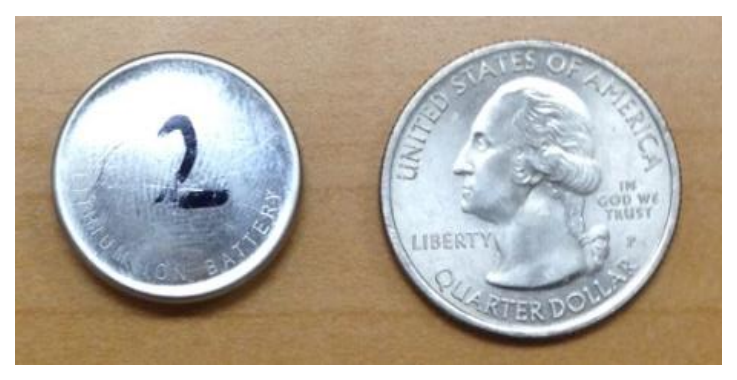

Figure 14. Supercapacitor coin cell.

\begin{tabular}{|c|c|}
\hline Capacitance (mF) & Discharge Time (s) \\
\hline 37 & 547 \\
\hline 91 & 1340 \\
\hline 470 & 6588 \\
\hline 540 & 7560 \\
\hline
\end{tabular}

Table 1. ASSIST custom supercapacitor discharge times. 


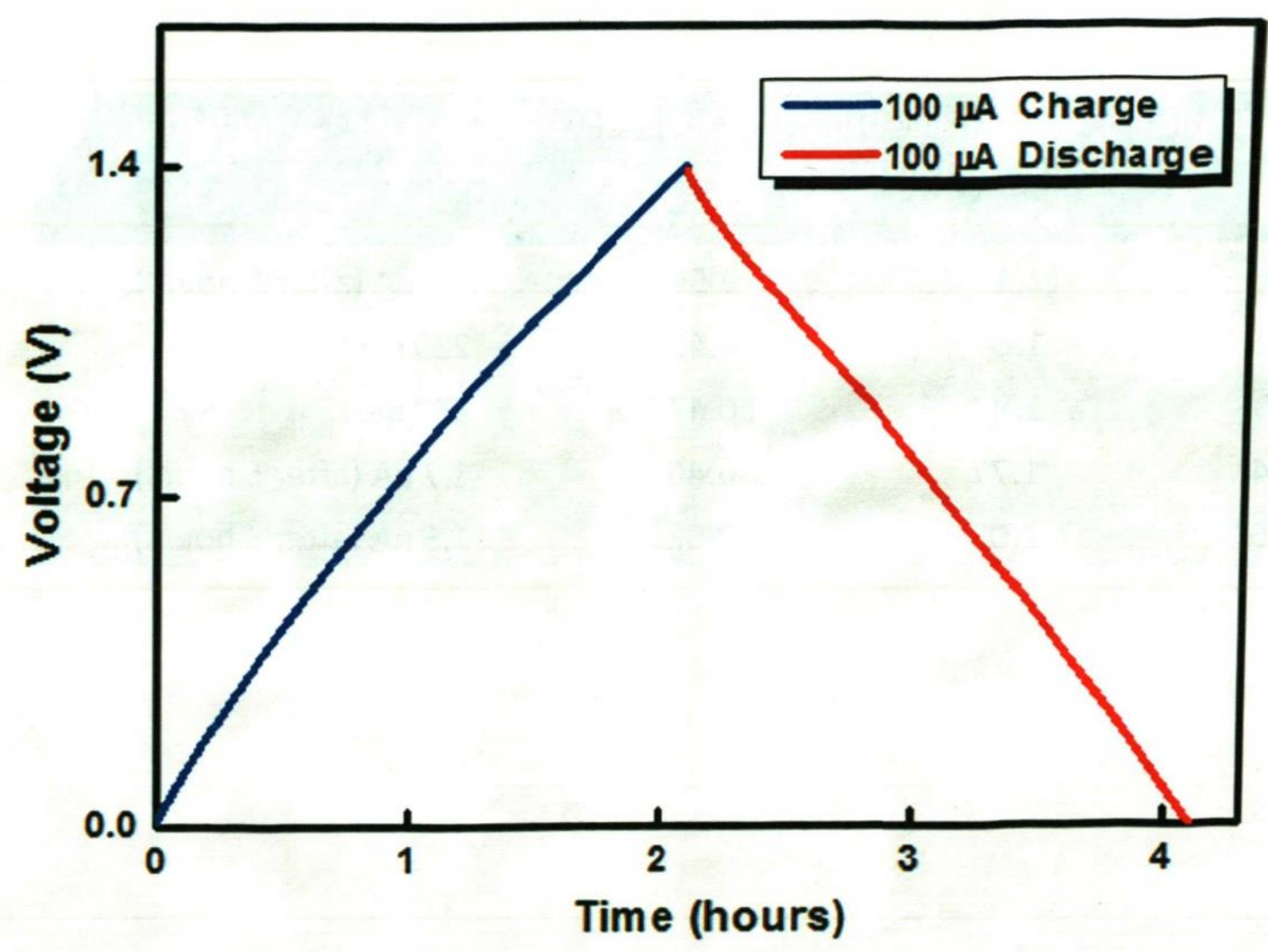

Figure 15. $540 \mathrm{mF}$ charge/discharge curve.

\section{LDO Regulator}

The LDO Regulator used in the SAP testbed is the TPS78326 from Texas Instruments [25]. It has an input voltage range of $2.75 \mathrm{~V}$ to $5.5 \mathrm{~V}$, an ultra-low quiescent current of $500 \mathrm{nA}$, a fixed output voltage of 2.6 $\mathrm{V}$, and an output load current of up to $150 \mathrm{~mA}$. The minimum input voltage is $2.75 \mathrm{~V}$ because the dropout of the regulator is about $150 \mathrm{mV}$. The reasoning behind why a $2.6 \mathrm{~V}$ rail is used to power the testbed is explained in section VI.B.

\section{Data Transmission}

The function of the Data Transmission block is to control the flow of the sensor data and transmit it to the smartphone and cloud server so that the user can view the data locally and the researchers and 
healthcare professionals can view the data remotely. BLE 4.2 is used as the wireless standard for transmitting the data, and this transmission is performed by a BLE SoC.

\section{BLE SoC}

The BLE SoC used in the SAP testbed is the DA14580 from Dialog Semiconductor [26]. The DA14580 has 32 general purpose digital I/O pins, a SPI interface, and 4 10-bit ADC channels. The SPI interface is used for the motion data from the accelerometer and one of the ADC channels is used for the ECG signal. The general purpose digital I/O pins and extra ADC channels provide flexibility to the SAP testbed in case additional sensors need to be added to improve the accuracy of the cardiac monitoring or the testbed needs to be used in another application. The DA14580 has other digital interfaces but they are not used in the SAP testbed.

For processing, the DA14580 uses a $16 \mathrm{MHz} 32$ bit ARM Cortex-M0 with a Serial Wire Debug (SWD) interface through which it is programmed in C by an external debugger. It also has a BLE core that is comprised of a Bluetooth baseband controller compatible with the BLE 4.2 specification. This controller is in charge of packet encoding/decoding and frame scheduling. It operates off of a $16 \mathrm{MHz}$ clock but also has a low power mode that supports a $32 \mathrm{kHz}$ clock. These clocks are generated by a $16 \mathrm{MHz}$ crystal, a $32 \mathrm{kHz}$ crystal, and their respective RC oscillators. For performing the RF part of the BLE protocol, the DA14580 uses a fully integrated $2.4 \mathrm{GHz}$ CMOS transceiver. It has a single ended RFIO interface that is $50 \mathrm{ohm}$ matched and a configurable transmission output power of $0 \mathrm{dBm}$ or $-20 \mathrm{dBm}$. The $-20 \mathrm{dBm}$ output power is used in the SAP testbed in order to consume less power during transmissions.

The DA14580 uses four internal memories. The first is an $84 \mathrm{kB}$ ROM that contains the BLE protocol stack as well as the boot code sequence for start-up. The second is a $32 \mathrm{kB}$ One-Time Programmable (OTP) memory that is used to store the application code as well as BLE profiles. The third is a $42 \mathrm{kB}$ 
system SRAM that is used for mirroring the application code from the OTP when the system wakes up from a sleep mode or powers up. The fourth is a series of four special low leakage SRAM cells known as retention RAMs used to store various data of the BLE protocol. These retention RAMs also store the processor stack when the system goes into Deep Sleep mode ensuring quick configuration of the BLE Core after the system wakes up.

The DA14580 operates off of a $0.9 \mathrm{~V}$ to $3.45 \mathrm{~V}$ rail and has a power management function integrated with a DC-DC converter configured in either Boost or Buck mode and separate LDO regulators for the different power domains of the system. When configured in Boost mode, the DC-DC converter acts as a step-up converter in order to provide power to the LDOs and can operate from $0.9 \mathrm{~V}$ to $2.0 \mathrm{~V}$. When configured in Buck mode, the DC-DC converter acts as a step-down converter and can operate from 2.35 V to 3.3 V. The DC-DC efficiency varies within the operating voltage ranges for each mode. This is shown in Figure 16. As shown in the figure, the DC-DC converter in Boost mode is most efficient at $1.5 \mathrm{~V}$ while in Buck mode it is most efficient at $2.35 \mathrm{~V}$. In the SAP testbed, the DC-DC converter is configured in Buck mode and is operated at $2.6 \mathrm{~V}$. Even though it is not the most efficient, it is operated at $2.6 \mathrm{~V}$ due to restrictions in the COTS LDO regulators available. This is discussed further in section VI.B. The reason for using Buck mode is that during the initial testing of the SAP testbed, it was discovered that the power consumption of the testbed in Boost mode was nearly twice of what it was in Buck mode. Since the power consumption was so high, the energy harvesters could no longer sustain the system. This behavior is not specified in the data sheet and was brought to the attention of Dialog Semiconductor, but because they did not have a solution to the problem, the system remained in Buck mode. Due to the testbed operating in Buck mode, the system shuts down when the voltage on the supercapacitor goes below $2 \mathrm{~V}$. Therefore, it is important to ensure that the power is properly managed so that this does not occur. 


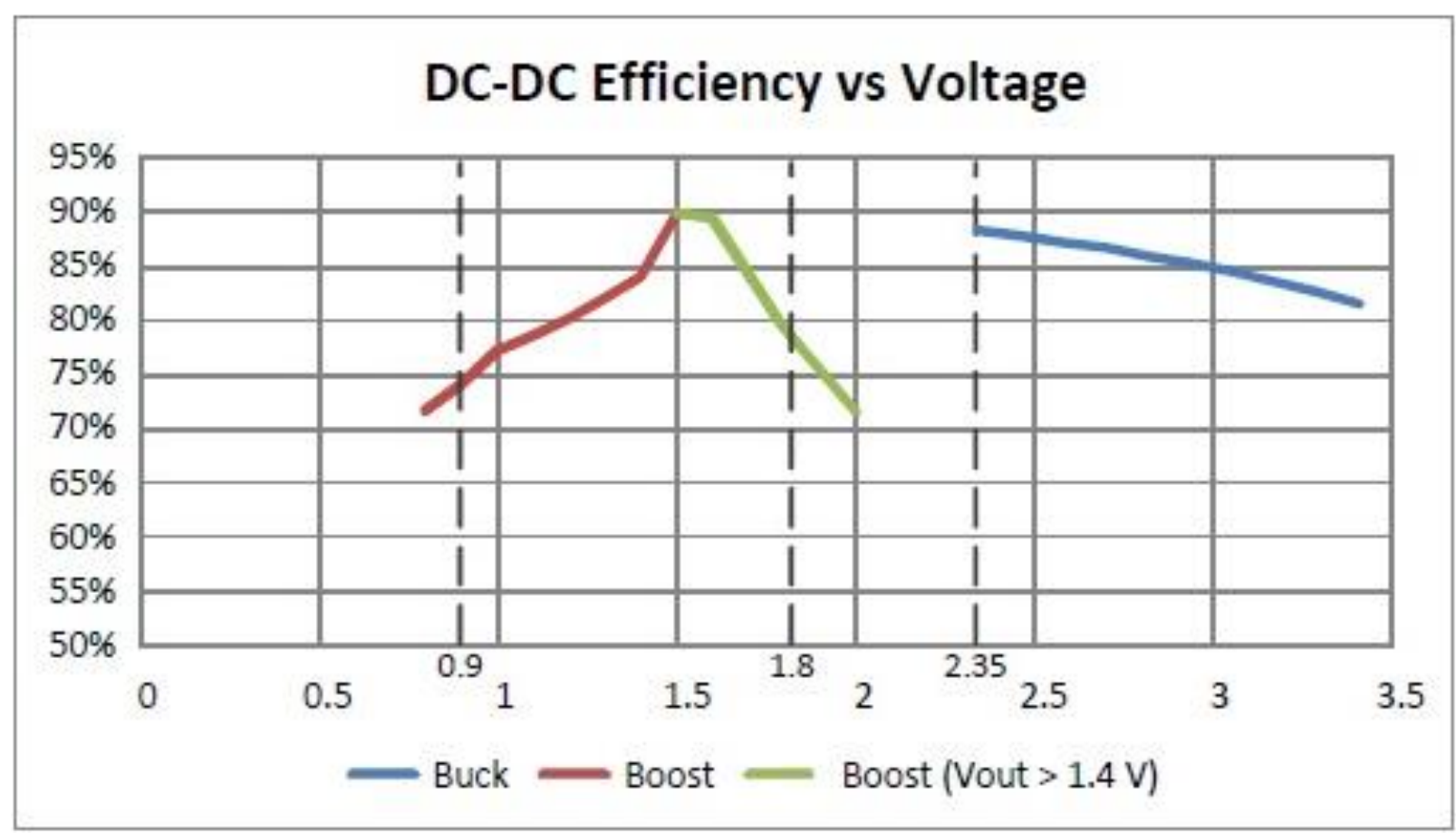

Figure 16. The BLE SoC's DC-DC Efficiency in Buck/Boost Mode at Various Voltage Levels.

In addition to the multiple power management configurations, the DA14580 has four different power modes: Active mode, Sleep mode, Extended Sleep mode, and Deep Sleep mode. In Active mode, the system operates at full speed. In Sleep mode, the ARM Cortex-MO is idle and waiting for an interrupt. In Extended Sleep mode, most power domains are off but the system SRAM retains its data. This means that no OTP mirroring is required upon waking up the system. In Deep Sleep mode, even more power domains are off including the system SRAM, so OTP mirroring is required when the system wakes up. The DA14580 consumes different amounts of power in these different modes with Active mode consuming the most. Section VI.C discusses how these power modes are utilized in order to minimize power consumption.

\section{Smartphone and Cloud Server}

The ECG and motion data is displayed locally using a smartphone and remotely using a cloud server. Currently, a custom Android application is used to display the data on the smartphone and therefore 
only an Android phone can be used. After the data is transmitted to the smartphone, the data is uploaded via WiFi to a time-series database that is hosted by Amazon. The view and display of the data is performed by a graphing service called Grafana. Figure 17 shows a screenshot of the Android application and the data displayed on the cloud server.

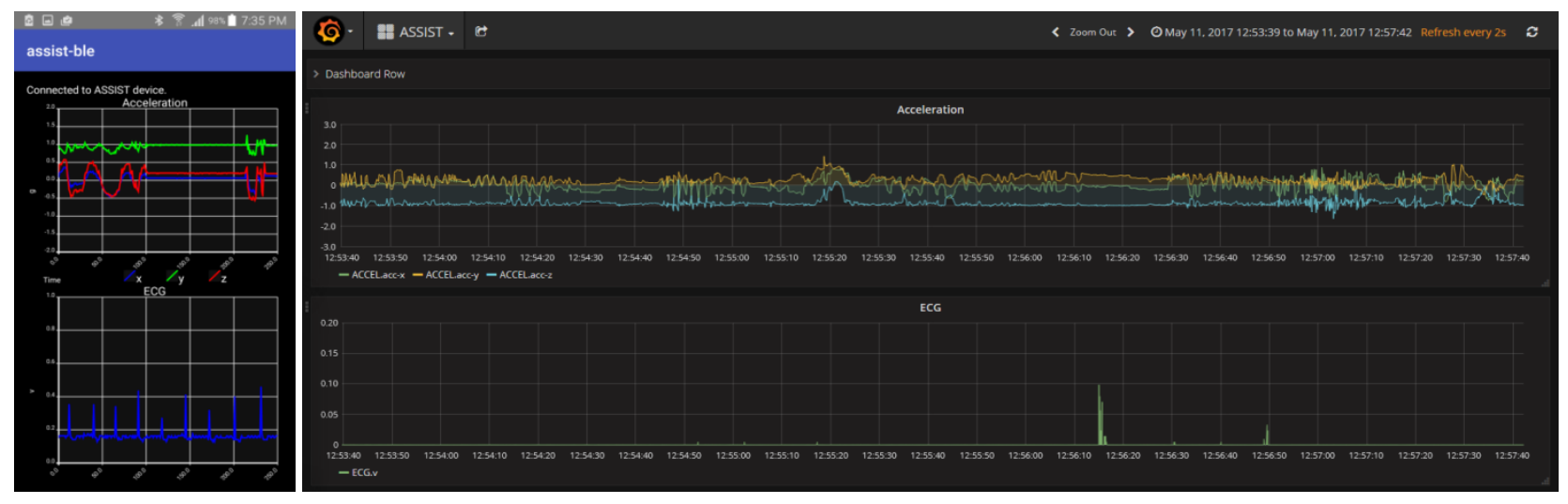

Figure 17. The Android application (left) and cloud server (right).

\section{E. SAP}

The SAP is a 1.1 by 0.9 inch PCB that contains the accelerometer, the ECG AFE, the LDO regulator, and the BLE SoC. It also contains a number of headers and an audio jack to interface with the other components in the SAP testbed. The audio jack interfaces with the ECG shirt, the JST header interfaces with the solar boost board, and the header pins contain extra I/O pins to interface with additional sensors and programming pins that interface with the external debugger. Figure 18 demonstrates the size of the SAP, while Figure 19 shows magnified front and back views of the SAP with markers indicating where each of the individual components are located on the PCB.

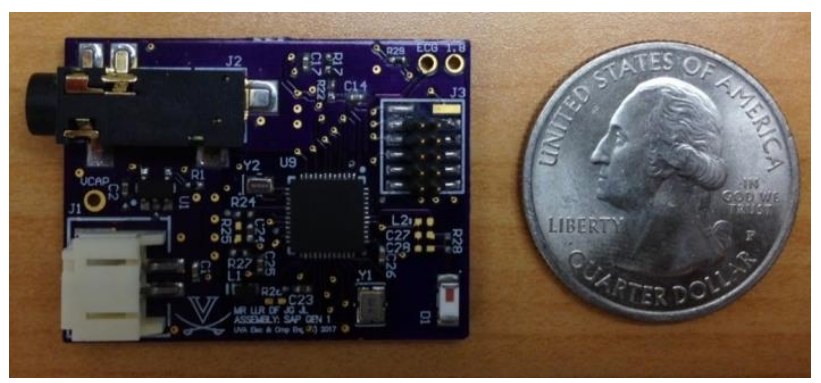

Figure 18. The size of the SAP. 


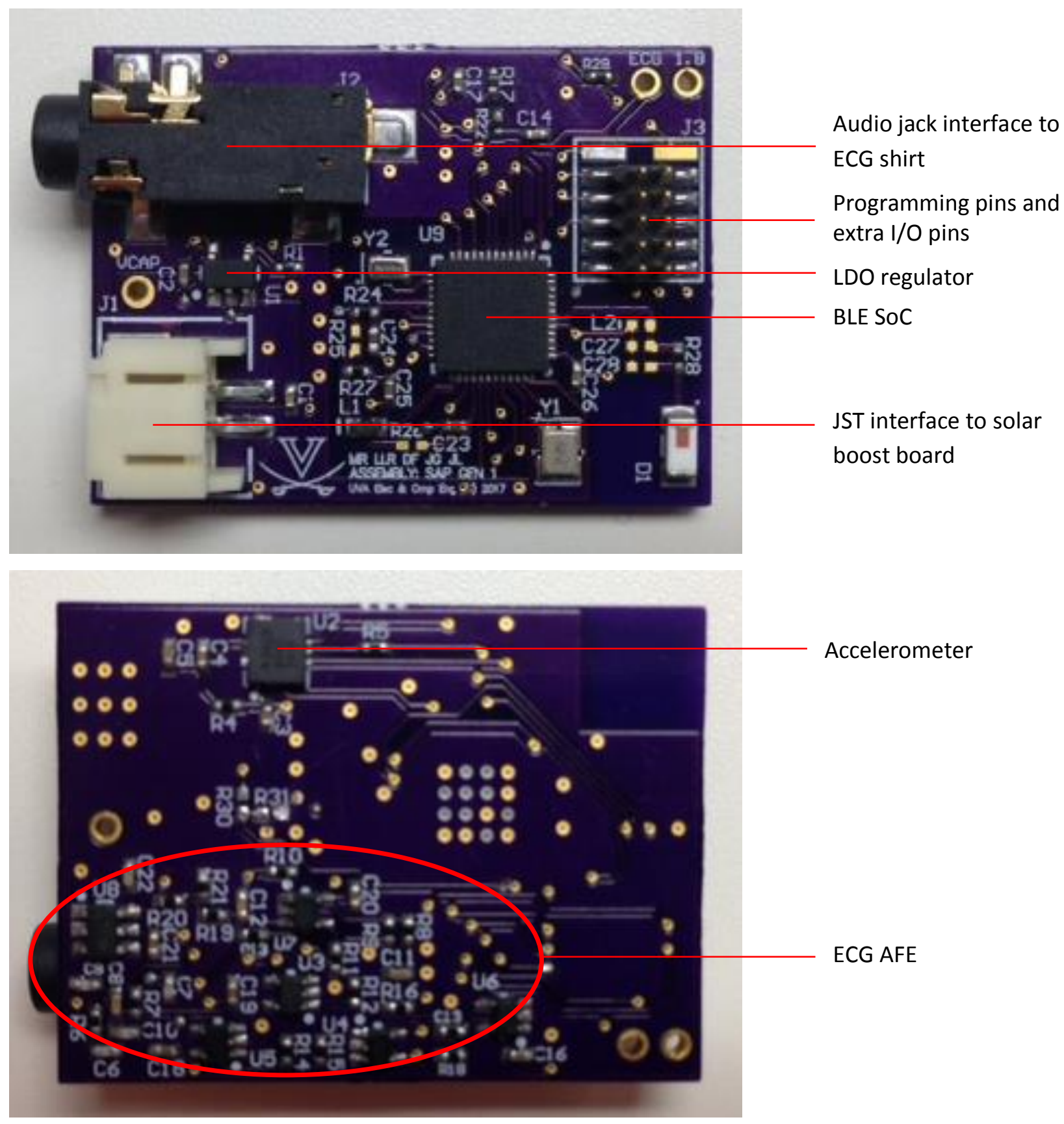

Figure 19. Magnified front (top) and back (bottom) views of the SAP. 


\section{Deployment Procedure, Power Consumption, and Results}

To date, the SAP testbed has not been deployed on CVD patients since it is not yet at a technology readiness level that permits such deployments. However, it has been deployed on researchers in order to evaluate its performance on conducting vigilant cardiac and activity monitoring over a specified period of time. It is important to note that because the vigilant monitoring of the testbed has only been evaluated on users with normal heart conditions in a laboratory environment, vigilance cannot be ensured for users in environments outside of the laboratory or for those with more extreme heart conditions such as atrial fibrillation. This section describes the procedure for deploying the SAP testbed on users, and it presents the power consumption of the testbed as well as the results that demonstrate that it is self-powered, wearable, and wireless, and can perform vigilant ECG and motion monitoring in the laboratory. Additionally, this section describes how the testbed can be modified in order to enable its cardiac and activity monitoring to be long-term.

\section{A. Deployment Procedure}

First, the ECG shirt is placed onto the intended user and an ECG cable is snapped onto the connection points in the front of the shirt. Next, the solar boost board is connected to the solar arm sleeve with a male-to-male JST connector. The supercapacitor is then placed onto the board and charged to $3 \mathrm{~V}$ using a power supply. With the power supply still connected to the supercapacitor, the output of the solar boost board is connected to the input of the SAP with a male-to-male JST connector. The power supply has to remain connected to the supercapacitor because the SAP draws milliamps of current when the BLE SoC is not programmed and this drains the supercapacitor very quickly. The SAP is then connected to the external debugger for programming. Once it is programmed, the external debugger is disconnected from the SAP and the power supply is disconnected from the supercapacitor. Next, the solar arm sleeve is slid onto the arm of the intended user and both the solar boost board and SAP are 
placed into the pocket. Finally, the ECG cable is connected to the SAP through the audio jack. After the SAP testbed is operational, the Android application on the smartphone is opened to view the data locally and the cloud server is accessed to view the data remotely.

\section{B. Power Consumption}

Table 2 shows the power consumption breakdown of the SAP testbed. It shows the measured power consumption of each of the major components of the testbed, as well as the power consumption of the testbed as a whole when performing motion monitoring alone and when performing ECG and motion monitoring.

\begin{tabular}{|c|c|}
\hline Component & Power $(\boldsymbol{\mu W})$ \\
\hline ECG AFE & 67 \\
\hline Accelerometer & 4.55 \\
\hline LDO Regulator & 9 \\
\hline BLE SoC Extended Sleep Mode & 9 \\
\hline BLE SoC Active Mode & 1598 \\
\hline Total System Power (motion (8.3 Hz) monitoring only) & 90 \\
\hline Total System Power (ECG (25 Hz) + motion (8.3 Hz) monitoring) & 370 \\
\hline
\end{tabular}

Table 2. Power consumption breakdown of the SAP testbed.

\section{Results}

The SAP testbed was deployed on several male subjects across different days. Each individual wore the system for multiple hours while doing their normal activities in the laboratory. During one of the deployments, the voltage on the supercapacitor (VCAP) and the intensity of the light on the solar cells were monitored for 4 hours to determine the impact of changes in illumination level on the energy 
available and the operation of the system. A $37 \mathrm{mF}$ supercapacitor was used in this deployment, and Figure 20 shows the results of the measurements.

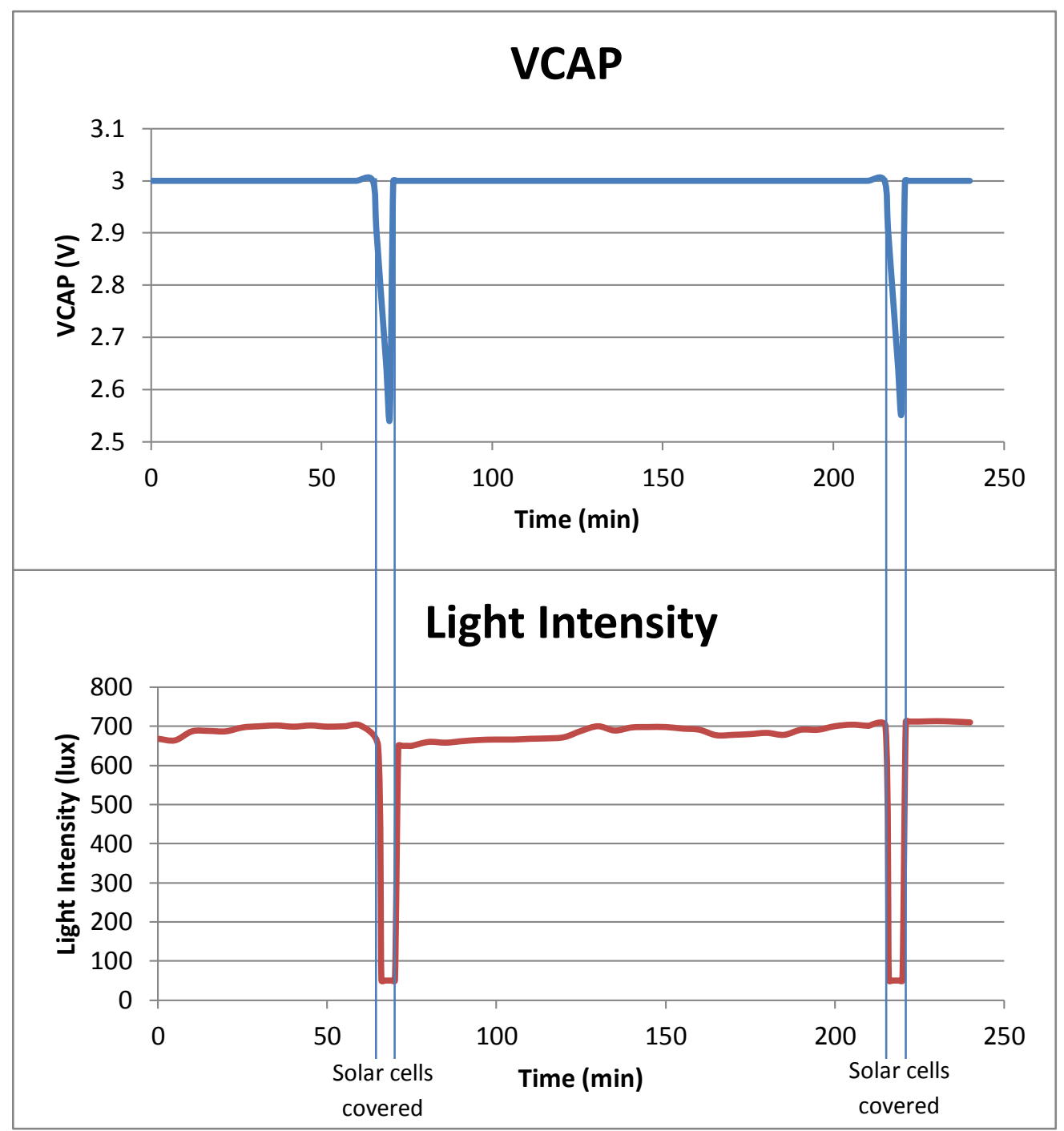

Figure 20. VCAP and light intensity over a 4 hour deployment.

As seen in the figure, VCAP always maintains its pre-charged voltage of $3 \mathrm{~V}$ unless the solar cells are fully covered at which point VCAP decreases at a rate of about $0.1 \mathrm{~V} / \mathrm{min}$. Once the solar cells are uncovered again, VCAP goes back up to $3 \mathrm{~V}$ within a few seconds. This behavior is expected since the power that the solar cells generate at around 700 lux is roughly $1.7 \mathrm{~mW}$, and after accounting for a DC-DC converter efficiency of $80 \%$, the power available to the system is $1.36 \mathrm{~mW}$ which is much greater than the $370 \mu \mathrm{W}$ 
that the system consumes on average. Similar behavior is expected when the light intensity is 500 lux or 250 lux since the power available to the system under those conditions is about $880 \mu \mathrm{W}$ and $480 \mu \mathrm{W}$ respectively.

In order to extend the characterization of the SAP testbed, the system was tested three times with a fully pre-charged supercapacitor as the only power source. The purpose of these tests was to ensure the continuous monitoring feature of the system necessary for vigilant sensing by determining the length of time that the system can run without any energy harvesting. It was important to ensure that the system can remain operational during this type of situation since there is no guarantee that the light intensity on the solar cells will always be high enough for sufficient harvesting during real-world deployments. The three capacitance values that the SAP testbed was tested with are $82 \mathrm{mF}, 164 \mathrm{mF}$, and $246 \mathrm{mF}$. These values are created from 1, 2, and $382 \mathrm{mF}$ COTS supercapacitors. During each test, the voltage on the supercapacitor was recorded until the system stopped running at $2 \mathrm{~V}$. The results of these tests are shown in Figure 21.

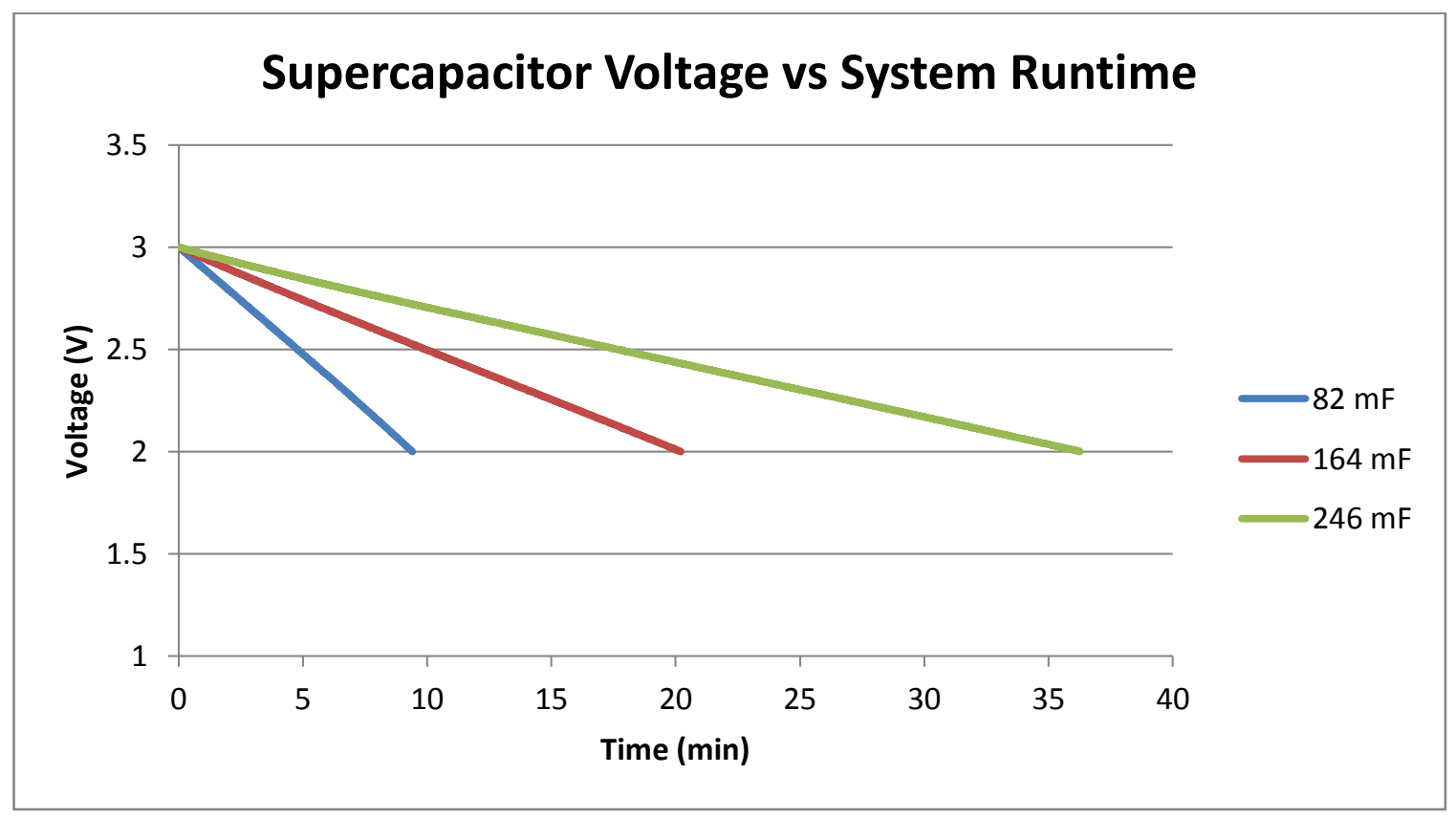

Figure 21. The results from running the SAP testbed on a fully pre-charged supercapacitor with no energy harvesting. 
This data is displayed differently in Figure 22, and it shows that there is a quasi-linear relationship between the size of the supercapacitor and the runtime of the system with no harvesting. Extrapolating to a $2 \mathrm{~F}$ supercapacitor suggests a non-harvesting operation duration of 8.7 hours, which is sufficient to sustain vigilant self-powered operation during long periods of time with no ambient light (e.g., sleeping), after which the supercapacitor will be re-charged due to the positive power balance in normal indoor lighting conditions.

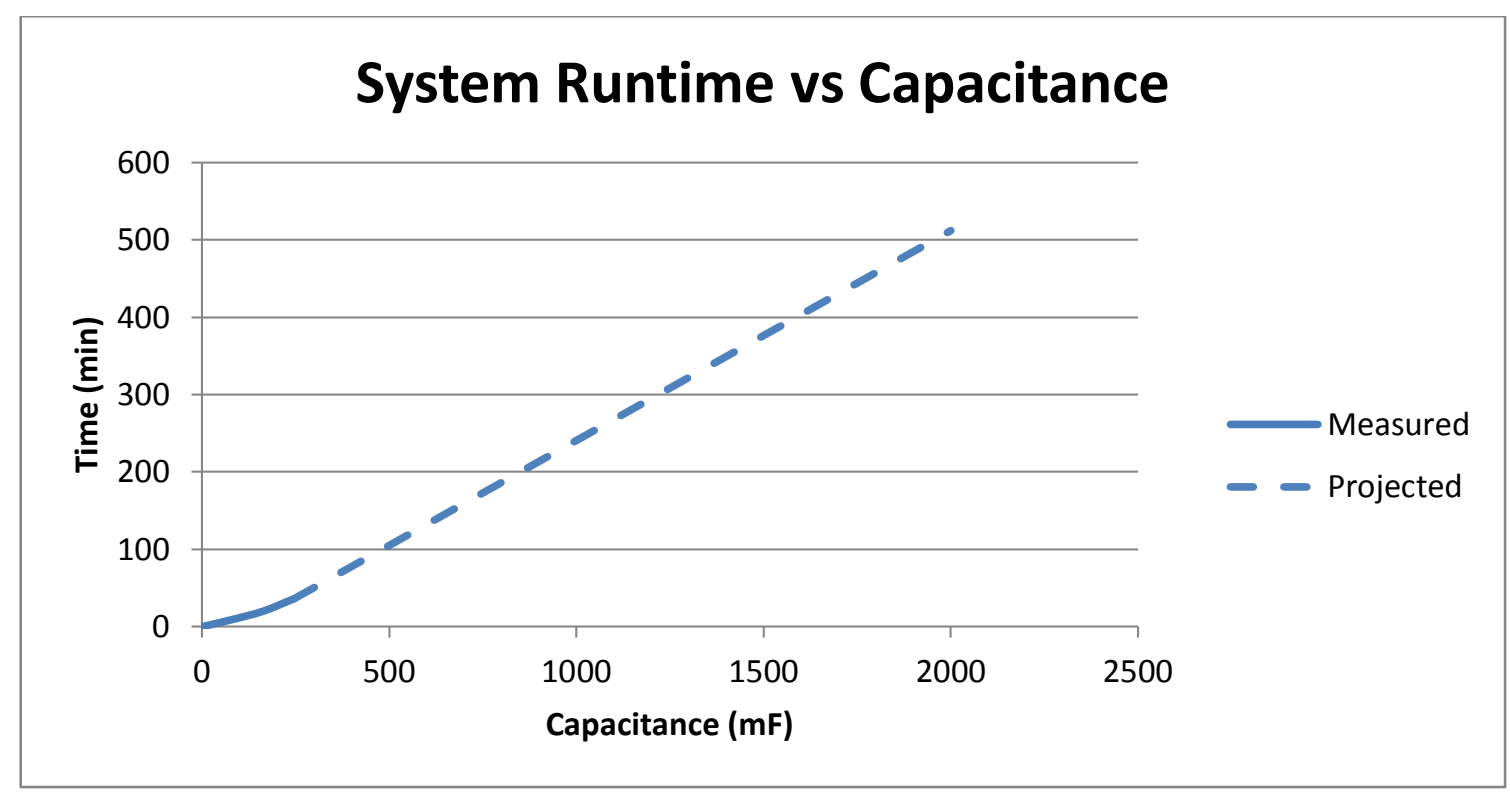

Figure 22. The measured and projected system runtime for different capacitance values.

The complete SAP testbed on the body and the Android application with real ECG and motion data measured at $25 \mathrm{~Hz}$ and $8.3 \mathrm{~Hz}$ respectively is shown in Figure 23. As seen in the application, small and large bodily movements are captured in the motion data and every QRS complex is captured in the ECG data. Since every required critical cardiac and activity event is captured and because the testbed is able to operate continuously for long periods of time with and without harvesting, the testbed successfully performs vigilant cardiac and activity monitoring for users with normal heart conditions in laboratory environments. 
With the SAP testbed integrated on the body like in Figure 23, the subjects neither reported any discomfort caused by wearing the system nor expressed difficulties in performing their normal activities during the different deployments. Therefore, the small form factor of the SAP testbed, the flexible solar cells, and the integration of the different components onto the arm sleeve accomplish the goal of wearability for the testbed.

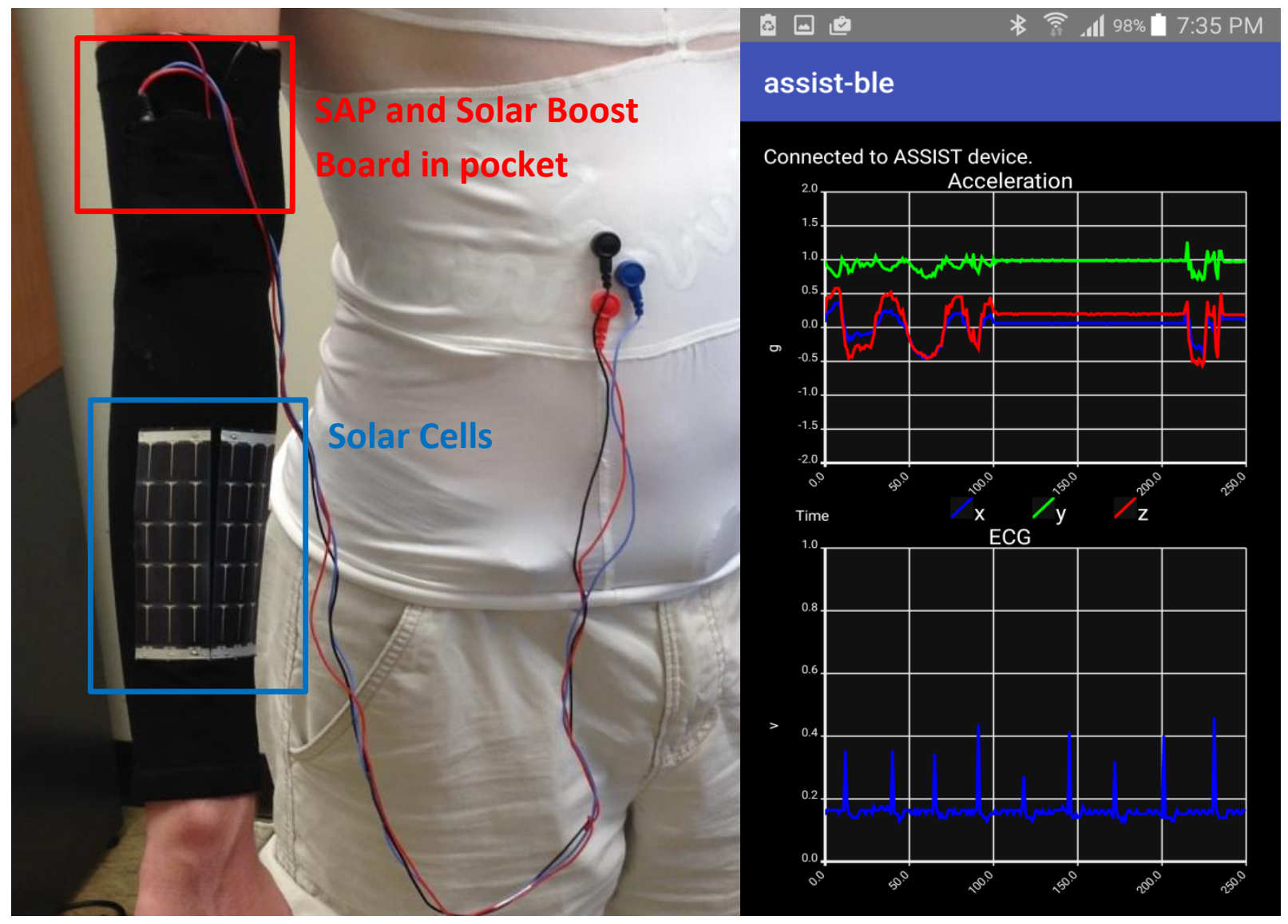

Figure 23. The SAP testbed on the body and the data displayed on the Android application.

\section{System Power Management}

Designing a wearable wireless BSN with multimodal vigilant sensing to have self-powered operation requires effective power management, and this is challenging for a number of reasons. The first reason is that multimodal sensing causes higher system power consumption due to the larger number of sensing modalities. The second is that the energy harvester must be small and compact in order for the 
BSN to be wearable, and depending on the type of harvester this can significantly limit the amount of power available to the system. The third is that wireless communication consumes a lot of power relative to the power that can be generated by the energy harvester. Finally, the fourth is that vigilant sensing requires higher sampling rates for the sensors and a high duty cycle for the data transmission which causes higher system power consumption. This thesis only focuses on these four reasons, although there are certainly many more.

High system power consumption and the limited power generated from the energy harvester make it difficult to ensure self-powered operation long-term, and therefore intelligent design decisions must be made in order to effectively manage system power. This section discusses the design considerations that can influence such decisions as they apply to the SAP testbed and self-powered wearable wireless multimodal vigilant sensing systems in general. In order to limit the scope of this discussion, design considerations that are not made in the SAP testbed are not mentioned. For example, a discussion on custom SoCs is not included because the testbed does not use a custom SoC.

\section{A. Managing Power Consumption in Multimodal Sensing}

When managing system power consumption in general, it is important to first determine the useable power available to the system. This is determined by calculating or estimating the average power that is generated from the energy harvester after any DC-DC converter inefficiencies. This number can then be used to establish a power budget, which is essentially the highest amount of power that the system can consume and still maintain self-powered operation. In wireless systems, wireless communication can take up a significant amount of this budget and sometimes even exceed it. Therefore, the data transmission in this communication must be duty cycled and the sampling rates of the sensors must be low in order to reduce this high power consumption to a level below the established budget. However, 
before figuring out how much duty cycling is needed and how low the sampling rates of the sensors have to be, it is first necessary to determine the power floor of the system.

The power floor refers to the amount of power that a system consumes when the duty cycle of the data transmission is $0 \%$ and therefore not transmitting. Perhaps the biggest contributors to the power floor are the components involved in the multimodal sensing of the system, since they always consume some amount of power regardless of whether or not data is being transmitted. These components include the sensors themselves and the components used to amplify, filter, or perform any other preprocessing on the signals from the sensors. Since the system must also have vigilant sensing, it is important for the data transmission to have a high duty cycle and for the sensors to have higher sampling rates. For this to be possible, the power floor must be minimized.

The sensing modalities in a BSN are entirely dependent on the application that it is designed for. Therefore, the power floor cannot simply be minimized by reducing the number of components and sensors. Instead, the best way to minimize the power floor is to use ultra-low power sensors and components. In the SAP testbed, the power budget is $480 \mu \mathrm{W}$ and the target power floor is $100 \mu \mathrm{W}$ so that most of the budget can be devoted to the BLE SoC. This target is met by using the ADXL362 3-axis digital output MEMS accelerometer to perform the motion monitoring and by using the LPV521 op-amp, LT6656 voltage reference, and general passives as the components that comprise the ECG AFE. Also, the TPS78326 is used as the regulator. While not a part of the multimodal sensing functionality, the regulator still contributes to the power floor since it is always active. All of these ultra-low power components operate at $2.6 \mathrm{~V}$ and have supply currents less than $5 \mu \mathrm{W}$. Because of this, the power floor in the SAP testbed is only $80.55 \mu \mathrm{W}$. Although different BSNs are bound to have different applications and thus different sensing modalities, the design considerations discussed here can still be used to make decisions that minimize the power floor and manage the power in multimodal sensing. 


\section{B. Managing Power Generated from a Wearable Energy Harvester}

A wearable energy harvester can significantly limit the amount of useable power available to a BSN. Therefore, this power must be carefully managed in order for it to sustain self-powered operation longterm. This can be accomplished through utilizing energy storage devices and regulators.

\section{Utilizing Energy Storage Devices}

Energy storage devices can be used to store excess energy generated by the energy harvester allowing the system to remain operational at times when there is little to no energy harvesting occurring. One common energy storage device is the supercapacitor. Supercapacitors can be made from various materials and have many different capacitance values which cause them to have different behaviors. Typically, supercapacitors with larger capacitances charge and discharge more slowly while supercapacitors with smaller capacitances charge and discharge more quickly. Therefore, it is important to weigh the costs and benefits of these behaviors and select a supercapacitor that will best meet the needs of the BSN. In the SAP testbed, a larger supercapacitor with a capacitance of $91 \mathrm{mF}$ or higher is used during standard deployments to ensure that the system can remain operational during long periods of time with no ambient light. However, sometimes a smaller supercapacitor with a capacitance of $37 \mathrm{mF}$ is used in order to demonstrate the capabilities of the solar cells by showing the quick charges and discharges of the supercapacitor when the solar cells are and are not covered.

\section{Utilizing Regulators}

Regulators are used to maintain constant voltage levels, and can help manage system power consumption by limiting the voltages that the other components in the system can operate off of. There are two common types of regulators that have different advantages and disadvantages: linear and switching. Linear regulators act like variable resistors and change their resistance in accordance with the load resulting in a constant output voltage. They can only step down their input voltage, and if the 
difference between the input and output voltages is high, the efficiency of the regulator becomes quite low since a lot of the power is wasted to heat. In contrast, switching regulators use an active device that switches on and off in order to maintain an average voltage value at the output. They can step up and step down their input voltage, and their efficiency is generally higher than that of linear regulators. However, there is a possibility of ripple on the output voltage due to the switching, and they also tend to consume more power due to a higher complexity.

Deciding on which type of regulator to use in a BSN entirely depends on the regulator features that are most important for the system to achieve self-powered operation. In the SAP testbed, these features are power consumption, output voltage, and output load current. Since COTS switching regulators tend to have higher power consumption than COTS linear regulators, a linear regulator is used in the system. In other BSNs, however, efficiency could be the most important feature or there could be a need to step up the input voltage. In these cases, a switching regulator would be more appropriate to use. After the type of regulator to use in the system is decided, the next step is to choose a specific regulator based on the other important features for the system. These features could include the quiescent current, output load current, output voltage, or form factor.

The TPS78326 is used in the SAP testbed as opposed to other linear regulators because of its ultra-low quiescent current of $500 \mathrm{nA}$, its fixed output voltage of $2.6 \mathrm{~V}$, and its max output load current of $150 \mathrm{~mA}$. The TPS78326 is a part of a family of regulators that have different fixed output voltages, and the $2.6 \mathrm{~V}$ regulator is used because it has the lowest output voltage in the family that is still within the required voltage range to configure the DC-DC converter in the BLE SoC into Buck mode. Having the lowest output voltage possible is important in order to minimize the power consumption of the components in the Sensing block. The ultra-low quiescent current is important in order to minimize the power consumption of the regulator. Finally, the $150 \mathrm{~mA}$ max output current is necessary in order to supply 
enough current to the system during Bluetooth transmissions and maintain the testbed's connection to the smartphone. All of the aforementioned specifications of the TPS78326 make it the ideal choice for the SAP testbed.

\section{Managing Power Consumption in Wireless Communication and Vigilant Sensing}

Wireless communication and vigilant sensing cause high power consumption in a BSN due to the high power cost of the data transmission and the high sampling rates of the sensors that vigilant sensing requires. Therefore, it is necessary to select a low-power wireless standard and SoC to transmit the data. It is also necessary to duty cycle the data transmission and select an optimum sampling rate that is high enough to achieve vigilant sensing but low enough as to not exceed the system's power budget. Modeling system power consumption can help determine this sampling rate. The discussion below assumes that a smartphone is used to display the sensor data and that only COTS SoCs are available.

\section{Selecting a Wireless Standard and System on Chip}

While there are many wireless standards to choose from, Bluetooth and BLE are easily the best options to use in a BSN due to their compatibility with most smartphones. Between Bluetooth and BLE, BLE is the better option since it consumes much less power. This is because it constantly remains in sleep mode until a connection is initiated, and the connection times are only a few milliseconds as opposed to the connection times of Bluetooth which are around 100 milliseconds [27]. The latest version of BLE, BLE 4.2 , is used in the SAP testbed for these very reasons.

Once a wireless standard is chosen, an SoC that performs the data transmission, controls the flow of data, and samples the sensors should be selected. There are many features of varying degrees of importance that SoCs have that must be considered in this selection process. These include the SoC's digital and analog interfaces, memories, processing power, power management, power consumption, 
radio transceiver, and software support. The DA14580 is the chosen BLE SoC for the SAP testbed because it is the COTS BLE SoC with the lowest power consumption available. In addition, it has multiple sleep modes that when duty cycled between its active mode, enable the average power consumption of the chip to be reduced to a level below the power budget of the system. Outside of the power realm, it has a SPI interface, a 4-channel 10-bit ADC channel, and up to 32 general purpose I/Os which make it compatible with most sensors. It also has many software packages that make it relatively simple to program the sampling rates of the sensors and the duty cycling of the data transmission.

\section{Duty Cycling the Data Transmission}

Performing data transmission with an SoC, even when using a low-power standard like BLE, consumes a lot of power relative to the other components in a BSN and the power that a wearable energy harvester can generate. This is demonstrated in the SAP testbed by the BLE SoC in active mode consuming nearly 20 times more power than the other components combined according to Table 2, and by consuming roughly $90 \%$ of the power that the solar cells can generate at 720 lux according to Figure 12 . For this reason, the data transmission in a self-powered BSN needs to be duty cycled. This duty cycling typically involves switching between the SoC's sleep and active operating modes, filling a buffer with the sensor data during active mode, and then transmitting that data buffer in packets over a connection event to wherever the data is being displayed. This process is illustrated in Figure 24.

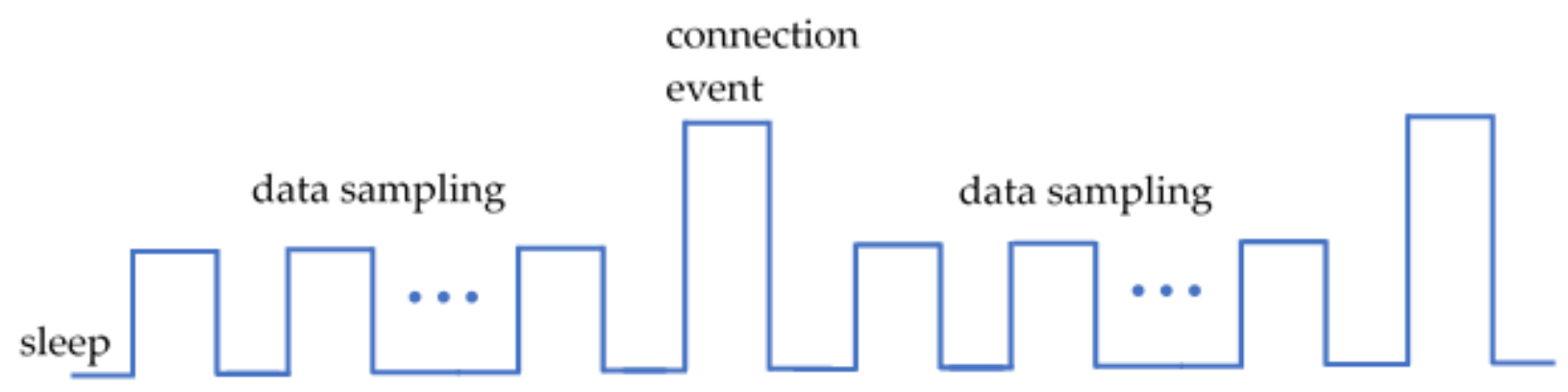

Figure 24. The BLE SoC duty cycling operation in the SAP testbed. 
In the SAP testbed, the BLE SoC wakes up from extended sleep mode and goes into active mode periodically to sample data from the sensors. Extended sleep mode is used as opposed to deep sleep mode because there is some overhead when the BLE SoC wakes up from deep sleep mode since it has to perform OTP mirroring. The sensor data is stored in an immutable 20 byte buffer, and once the buffer is full, packets containing the data are sent in a connection event to the smartphone. This means that, for the SAP testbed and similar wireless systems, the sampling rate of the sensors controls the rate at which the buffer is filled, the transmission rate of the system, and the rate at which the BLE SoC is going in and out of sleep mode. Therefore, the sampling rate also controls the average power consumption of the SoC.

\section{Modeling Power Consumption and Selecting an Optimum Sampling Rate}

Given that the sampling rate of the sensors controls the SoC's average power consumption, it is necessary to select an optimum sampling rate that ensures both self-powered operation and vigilant sensing for the BSN. Creating a model for the power consumption of the system can help project system power at different sampling rates and determine the optimum rate. Total system power consumption can be modeled by the following equation:

$$
\mathrm{P}_{\text {tot }}=\mathrm{P}_{\mathrm{cmpts}}+\mathrm{P}_{\mathrm{SoC}}
$$

$P_{\text {tot }}$ represents the total system power consumption, $P_{\text {cmpts }}$ represents the power consumption of all components in the system excluding the SoC (i.e. the power floor), and $\mathrm{P}_{\mathrm{Soc}}$ represents the power consumption of the SoC. In the SAP testbed, $\mathrm{P}_{\text {cmpts }}$ includes the power consumption of the accelerometer, the LDO regulator, and the ECG AFE, while $\mathrm{P}_{\text {Soc }}$ just includes the power consumption of the BLE SoC. Since the SoC's data transmission must be duty cycled, it is necessary to consider the time that the SoC spends in its different operating modes and how much power each mode consumes. Three 
common modes that SoCs cycle through include sleep, active and sampling, and active and transmitting. Therefore, $\mathrm{P}_{\text {Soc }}$ can be modeled by the following equation:

$$
\mathrm{P}_{\mathrm{SoC}}=\frac{\mathrm{fT}_{\mathrm{c}} \mathrm{T}_{\mathrm{as}} \mathrm{P}_{\mathrm{as}}+\mathrm{T}_{\mathrm{at}} \mathrm{P}_{\mathrm{at}}+\left(\mathrm{T}_{\mathrm{c}}-\mathrm{T}_{\mathrm{at}}-\mathrm{fT}_{\mathrm{c}} \mathrm{T}_{\mathrm{as}}\right) \mathrm{P}_{\mathrm{s}}}{\mathrm{T}_{\mathrm{c}}}
$$

$T_{c}$ represents the length of the connection interval, $T_{\text {as }}$ represents the period of time when the BLE SoC is active and sampling the sensor data, and $T_{\text {at }}$ represents the transmission period. The power consumption of the SoC active and sampling, active and transmitting, and sleeping are represented by $P_{a s}, P_{a t}$, and $P_{s}$ respectively. Finally, $f$ represents the sampling rate of the sensors.

The first part of the equation, $\mathrm{f}^{*} \mathrm{~T}_{\mathrm{as}}{ }^{*} \mathrm{P}_{\mathrm{as}}$, represents the power consumed when the SoC is in active mode and sampling the sensor data. The second part of the equation, $\left(T_{a t} / T_{C}\right) * P_{a t}$, represents the power consumed when the SoC is in active mode and transmitting the data to the smartphone over the connection interval. The last part of the equation, $\left(1-T_{a t} / T_{C}-f * T_{a s}\right) * P_{s}$, represents the power consumed when the SoC is in sleep mode. The SoC is only in sleep mode at times when it is not in active mode. Since this power model uses parameters that are not specific to a single wireless standard, they can be used to project system power consumption for a variety of wireless BSNs.

In order to accurately project system power consumption using a power model, measurements or estimates for the parameters within the model must first be made. The parameters for the BLE SoC's data transmission in the SAP testbed were measured using the Smart Snippets tool from Dialog Semiconductor that comes with the evaluation kit for the DA14580. This tool measured the current drawn by the BLE SoC in each of its operating modes during its duty cycling which allowed for accurate measurements for each parameter in the power model to be made. Figure 25 shows a screenshot of these measurements. The measured parameters in combination with the power model were used to project the system power consumption of the SAP testbed. This projection is shown in Figure 26, and is 
quite accurate given that it projects a power consumption of around $400 \mathrm{uW}$ at $25 \mathrm{~Hz}$ when in reality, the power consumption is $370 \mathrm{uW}$ at $25 \mathrm{~Hz}$.

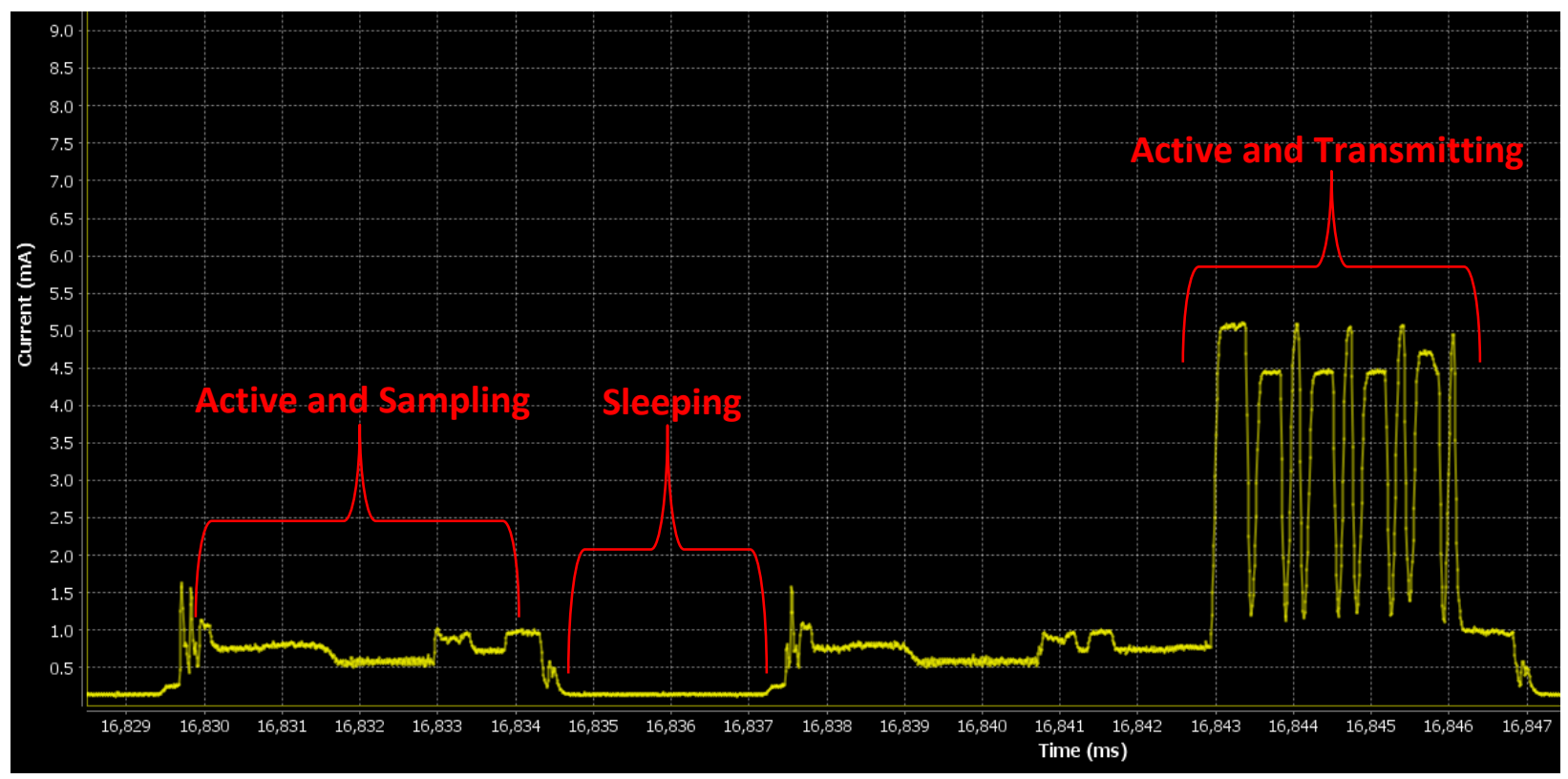

Figure 25. The current drawn during the BLE SoC's duty cycling operation in the SAP testbed.

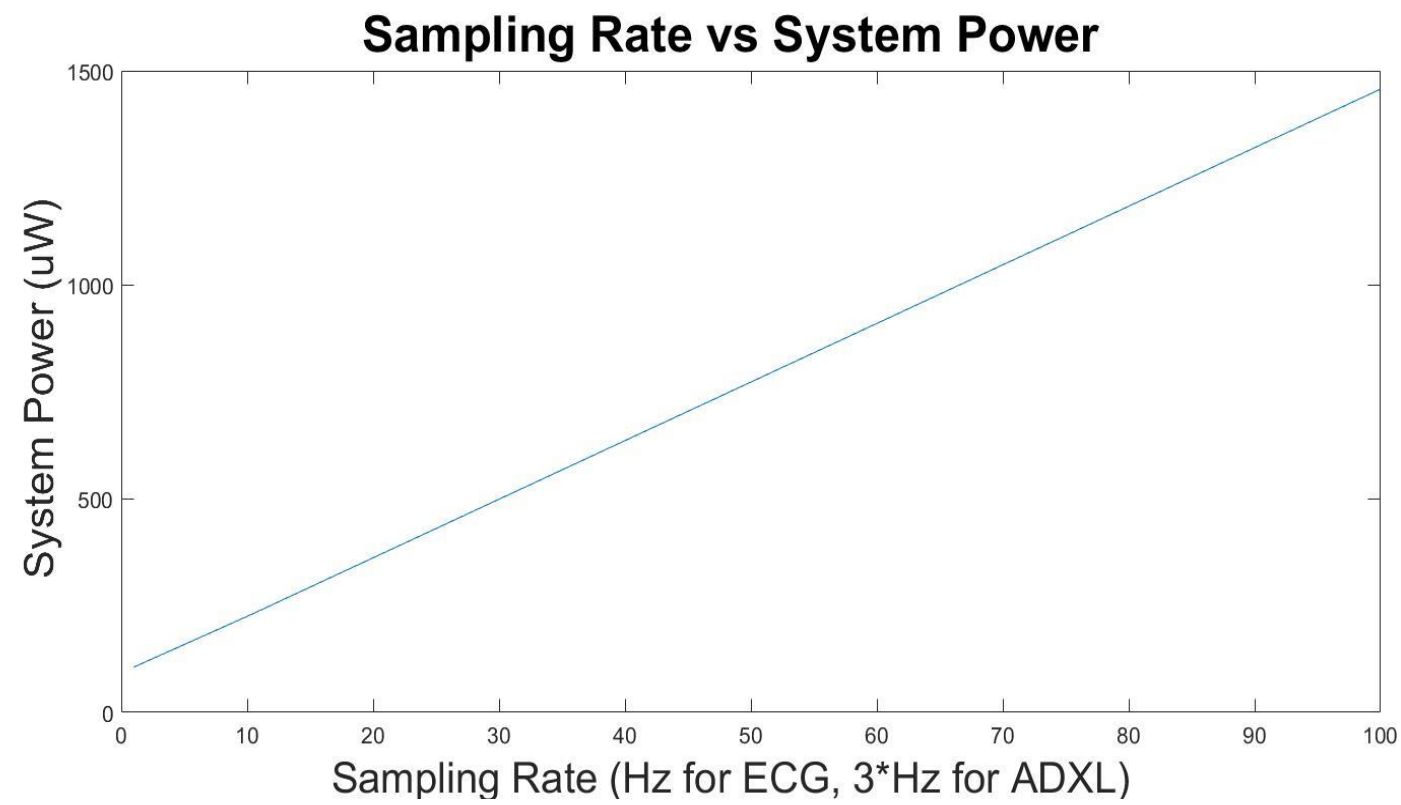

Figure 26. Projected system power for the SAP testbed based on the power model for different sampling rates. ECG is sampled at 3 times the rate of the accelerometer (ADXL). 
As shown in Figure 26, system power consumption increases linearly with sampling rate and therefore decreasing the sampling rate can reduce the power consumption. However, when vigilant sensing is required, it is necessary for the sampling rate to be high enough in order to capture all critical events. In the SAP testbed, these critical events include an irregular heart rate and bodily movements. Therefore, the sampling rate must be high enough to capture all QRS complexes in the ECG signal as well as small and large movements detected by the accelerometer. Using Figure 26, the sampling rate must be $30 \mathrm{~Hz}$ or below since the power budget of the SAP testbed is $480 \mu \mathrm{W}$. After some experimentation with sampling rates below $30 \mathrm{~Hz}$, it was determined that $25 \mathrm{~Hz}$ is the lowest sampling rate that can capture all QRS complexes in the ECG signal. It was also determined that a much smaller sampling rate can be used to detect bodily movements since this motion occurs over a longer period of time than the QRS complex in an ECG signal. Therefore, $25 \mathrm{~Hz}$ is used to sample ECG and $8.3 \mathrm{~Hz}$ (one third of $25 \mathrm{~Hz}$ ) is used to sample motion. Figure 27 shows the ECG signal at $20 \mathrm{~Hz}$ and $25 \mathrm{~Hz}$ to show the difference that the sampling rate makes in the quality of the signal. The $20 \mathrm{~Hz}$ signal misses a few R peaks while the $25 \mathrm{~Hz}$ signal misses none. It is important to note that these ECG signals are measured from a subject with normal heat conditions. Therefore, $25 \mathrm{~Hz}$ may not be a sufficient sampling rate to capture all QRS complexes in subjects with more extreme heart conditions such as atrial fibrillation.
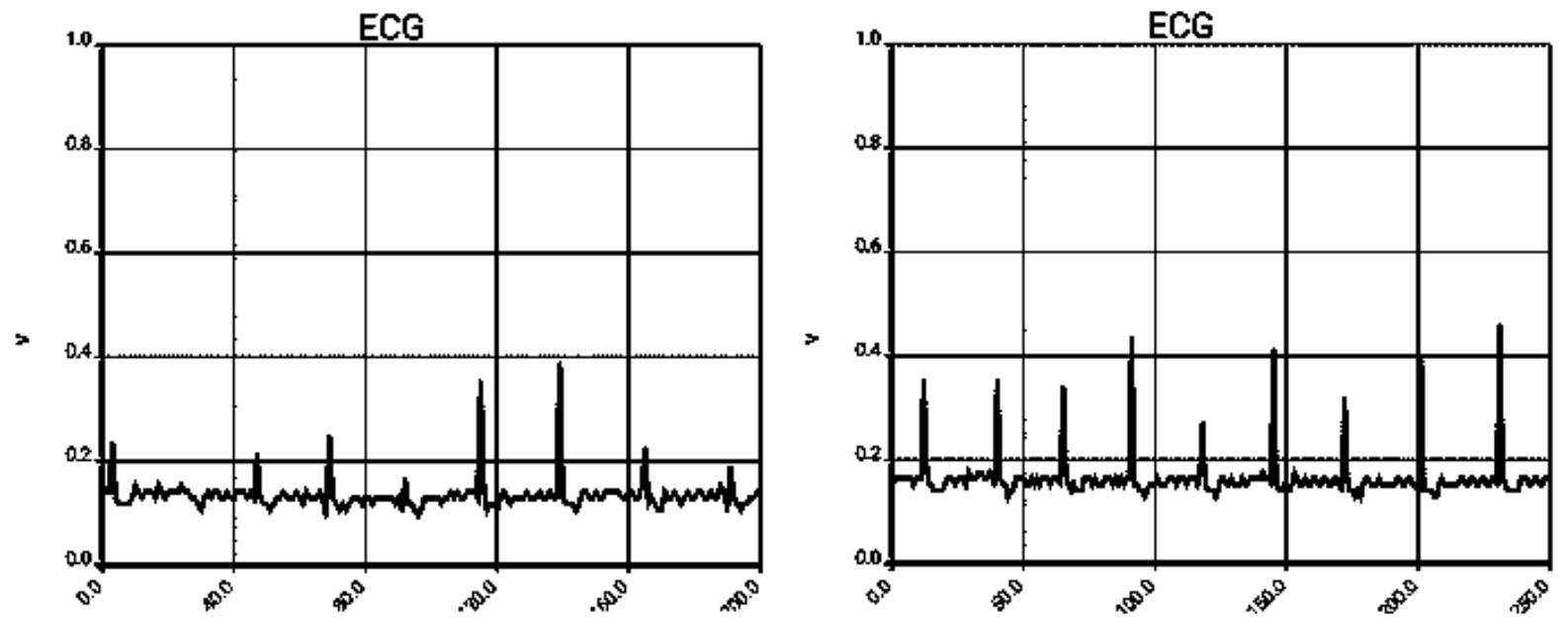

Figure 27. ECG sampled at $20 \mathrm{~Hz}$ (left) and $25 \mathrm{~Hz}$ (right) 


\section{Energy Harvesting and System Wearability Optimization}

In order for a BSN to achieve self-powered operation long-term, the average power generated from the energy harvester must be greater than the average power that the system consumes. Therefore, it is necessary to optimize the energy harvesting. However, for several types of harvesters, the size of the harvester directly impacts the wearability of the system as well as the amount of power generated. This fact makes optimizing both the energy harvesting and system wearability for these types of harvesters quite challenging since a larger harvester means more power generated but less wearability while a small harvester means less power generated but more wearability. This section discusses the design considerations involved in optimizing energy harvesting and system wearability as they apply to the SAP testbed and self-powered wearable wireless multimodal vigilant sensing systems in general. The discussion on energy harvesting optimization focuses only on solar and thermoelectric energy harvesters in order to limit its scope.

\section{A. Energy Harvesting Optimization}

Solar and thermoelectric are two common types of energy harvesting where the size of the harvester can directly impact the amount of power available to the system. This energy harvesting can be optimized by choosing a location on the body to place the harvester that maximizes harvesting and by utilizing an efficient DC-DC converter that has maximum power point tracking.

\section{Optimizing Solar Energy Harvesting}

Solar energy harvesting is a process in which the energy of light is converted into electricity by an electrical device called a solar cell. Solar cells are made from a variety of photovoltaic materials that can harvest energy from high light intensity conditions like sunlight to low light intensity conditions like artificial light, with some materials being better at harvesting energy under low light intensity conditions than others. In self-powered BSN design, it is important to use solar cells that are designed to harvest 
energy more efficiently under the light conditions that the BSN is most likely to be used in. It is also important to use solar cells that have a power per unit area under those light conditions such that only a small number of small cells would be able to harvest enough energy to power the system long-term. The cells must be as small as possible and there must be as few of them as possible so that they can be comfortably placed on the body and are not a burden to the user.

Solar energy harvesting can be optimized by maximizing the level of light intensity on the solar cells. Since it is not always possible to control the source of light and it is not feasible to constantly shine a light on the cells, this can be accomplished by placing the solar cells in a location on the body where the most light will be. These locations include the head, neck, shoulders, and arms. However, placing solar cells on the head or neck of the user would most likely burden them, so the two best locations for this placement are the shoulders and arms.

\section{Optimizing Thermoelectric Energy Harvesting}

Thermoelectric energy harvesting is a process in which a thermoelectric generator (TEG) harvests the temperature gradient across the device and converts it into electricity. In self-powered wearable BSNs, TEGs are placed on the body, so this gradient is the difference between the skin temperature and the ambient temperature. This difference is typically only $10^{\circ} \mathrm{C}$ or less and therefore the TEGs selected for the system must be very efficient at harvesting small gradients [28]. They must also have a good heat sink because during the energy harvesting, the entire TEG rises in temperature until it reaches equilibrium with the equipment housing which means that the gradient and thus the harvested energy is negligible. With a heat sink, the TEG can better maintain a constant temperature gradient over long periods of time. In addition, the total area of the selected TEGs must be large enough to power the system long-term. This area can come from just a few larger TEGs or many smaller TEGs. However, like 
solar cells, the TEGs must be as small as possible and there must be as few of them as possible in order to optimize the wearability of the system.

Thermoelectric energy harvesting can be optimized by maximizing the temperature gradient on the TEGs. This can be accomplished by placing the TEGs in a warmer area on the body and by cooling the sides of the TEGs that have the heat sink and are not on the body. Since it is not feasible to constantly hold a cold pack on the TEGs to cool them, the best method of cooling is to place the TEGs in a location on the body that gets a lot of airflow. The location on the body that is both warm and has a lot of airflow is the upper arm. This is because this area is close to the armpit, which is a particularly warm area of the body, and arms frequently move around which gives them good airflow.

\section{Utilizing DC-DC Converters}

Solar and thermoelectric energy harvesting can also be optimized through utilizing a DC-DC converter with maximum power point tracking (MPPT). DC-DC converters are used to step up voltage, and they are necessary to use at the output of an energy harvester when the harvester output voltage is below the level that the rest of the system is required to operate at. A DC-DC converter with MPPT can optimize energy harvesting by extracting the maximum power available from the harvesters. In addition to MPPT, it is important for the DC-DC converter to have a high efficiency. High efficiency is important in order to maximize the useable power available to the system. In fact, efficiency must be taken into account when selecting how many solar cells or TEGs to use in the system. For example, if a system consumes $190 \mu \mathrm{W}$, the solar cells generate $200 \mu \mathrm{W}$, and the DC-DC converter efficiency is $80 \%$, then the power available to the system is only $160 \mu \mathrm{W}$ and therefore more solar cells need to be added to sustain the system.

\section{Optimizing Energy Harvesting in the SAP Testbed}

Solar energy harvesting is used in the SAP testbed because COTS solar cells can generally harvest more energy than COTS TEGs in a smaller and more flexible form factor [29]. The MP3-37 flexible solar cells 
from Power Film Solar are used to perform this harvesting because they have a high power per unit area and can easily conform to the body despite having a large surface area of $41.61 \mathrm{~cm}^{2}$. The number of solar cells used in the SAP testbed was determined through measuring the power that the solar cells generate at different indoor illumination levels of 250 lux, 500 lux, and 720 lux. At the same time this decision was made, the BQ25504 Ultra Low-Power Boost Converter was chosen to be used in the system since it has MPPT, a high efficiency between $80 \%$ and $90 \%$, and a cold start voltage below the output voltage of the solar cells. Using the $80 \%$ efficiency number and the characterization of two of the MP337 solar cells shown in Figure 12, it was determined that two solar cells would be sufficient to sustain the system long-term. The characterization in Figure 12 was also used to determine that the MPPT in the DC-DC converter would have to be programmed to about $60-70 \%$ of the maximum output voltage in order to extract the maximum power available from the solar cells. These solar cells were then sewn into an armband in order to maximize the light intensity seen on the cells and to optimize the wearability of the system.

\section{B. System Wearability Optimization}

User preferences need to be taken into account when designing a BSN to be wearable in order for it to not be a burden to the user both inside and outside of the laboratory. Although system wearability is subjective, users generally prefer that BSNs are compact, embedded on the body, and do not affect their daily behavior [3]. Therefore, system wearability can be optimized by designing a BSN to have these qualities. However, this can be challenging when a BSN must also have self-powered operation and multimodal sensing since it would require an energy harvester on the body and multiple sensors which can make the system quite bulky. This challenge can be addressed by minimizing the size of the energy harvester (discussed in section VII.A), matching the sensing locations to the location of the energy 
harvester, integrating most of the system components onto small printed circuit boards, and by integrating sensors into textiles.

\section{Matching a Sensing Location with an Energy Harvesting Location}

As previously discussed, energy harvesting optimization often involves choosing a specific location on the body to place the harvester. Therefore, in order to keep the system compact, it is important to place the sensors as close to the harvester as possible. However, this is easier to do with some sensors than it is with others. Ozone sensors, for example, have a small form factor and do not require a specific location on the body, so they can easily be placed close to the energy harvester [14]. Motion sensors like accelerometers also have a small form factor, but they sometimes require placement in specific locations on the body depending on the application of the BSN. If this is the case, then it may be more feasible to match the energy harvesting location with the sensing location or even change the energy harvester to one that can better harvest energy at the location where the motion sensor must be placed. For example, if an accelerometer must be placed on the user's ankle and the intended energy to be harvested is solar, then it may not be practical to move the solar cells closer to the ankle since they would harvest much less energy. So instead, it could be better to use piezoelectric energy harvesting since that harvester could be more easily integrated into a shoe like in [13]. Finally, ECG electrodes are more difficult to match to an energy harvesting location since they are not compact and require specific placement on the body. However, since they tend not to be directly integrated onto a PCB like motion sensors and ozone sensors, longer ECG cables can be used to help match them to the location of the energy harvester.

In the SAP testbed, the accelerometer is integrated onto the SAP. Since the location of it does not matter for the motion sensing, the SAP is matched to the location of the solar cells by placing it into the sewn-in pocket on the solar arm sleeve. The ECG shirt is matched to the location of the solar cells by 
threading the ECG cable through the top of the shirt and then through the right-hand arm sleeve and the connecting it to the SAP through the audio jack. In matching these sensing locations to the harvesting location, the SAP testbed is compact and embedded on the body.

\section{Integrating System Components onto PCBs}

Another way to ensure that a BSN is compact is by integrating as many of its components as possible onto a small PCB. If multiple PCBs are required like in the SAP testbed, then a case to hold all of the PCBs could be designed using a 3D printer. Also, if the resources are available, flexible PCBs or even PCBs integrated into textiles like in [30] could be considered in order to further improve system wearability.

\section{Integrating Sensors into Textiles}

Sensors can also be integrated into textiles in order to improve system wearability. One example of this is the ECG shirt in the SAP testbed. Despite it being slightly more difficult to get the electrodes to establish a connection than with traditional electrodes, the ECG shirt is far more wearable. This is because traditional electrodes can be very uncomfortable, difficult to place correctly for those not familiar with ECG, cause rashes for those with sensitive skin, time consuming to take on and off, and painful to remove for those with hairy chests. In contrast, the ECG shirt is much more comfortable due to its soft and conforming fabric and easiness to take on and off.

\section{System Flexibility and Modularity Optimization}

Real-world deployments for a BSN can be unpredictable and its functional requirements can change as new information is acquired during research. Therefore, it is important to optimize its flexibility and modularity. However, this can be challenging given how many potential applications there are to consider during this optimization. Some of the ways that this can be addressed include designing the BSN to be compatible with different energy harvesters, enabling it to have sensing modalities added or removed, and designing its architecture in such a way that enables older technology to be easily 
replaced with newer technology when it becomes available. There are other ways to address this challenge, of course, but these are the ones most relevant to the SAP testbed and are therefore the only ones discussed here.

When designing a BSN to be compatible with different energy harvesters, it is necessary to clearly define the interface between each potential harvester and the rest of the system, and determine what DC-DC converter and external circuitry is needed to accompany each harvester. Next, it is important to determine where these components should be located in the system. Since different energy harvesters often require different DC-DC converters and external circuitry, it is best to try to separate the energy harvesting circuitry for each harvester from each other and the rest of the system. This is accomplished in the SAP testbed by integrating the energy harvesting components for each potential harvester onto separate PCBs with the interface between each $P C B$ and the SAP being the output of the DC-DC converter. One of these PCBs in the SAP testbed is the solar boost board discussed back in section IV.B. Another is called the TEG boost board and it is designed so that the testbed can be compatible with TEGs. Essentially, the SAP testbed is compatible with any energy harvester as long as a 'boost board' with a DC output is designed to accompany it.

When designing a BSN to be able to have sensing modalities added or removed, it is necessary to select an SoC that has many different types of interfaces that can accommodate a multitude of sensors and to ensure that these interfaces are made available on the PCB that the SoC is integrated onto. The DA14580 in the SAP testbed is one such SoC that has many different types of interfaces. It has 32 general purpose digital I/O (GPIO) pins, a SPI interface, an I2C bus, 2 UARTs, and 4 10-bit ADC channels. The SPI interface and one of the ADC channels is used for the motion data and the ECG data respectively. In order for the testbed to be able to have sensing modalities added to it, two extra ADC channels and four extra GPIO pins are made available on the SAP through header pins. Removing 
sensing modalities on the SAP testbed simply requires that it be programmed to ignore certain interfaces when sampling and transmitting the data.

Since electronics technology is rapidly evolving and designs frequently change, it is important to design a BSN's architecture in such a way as to allow older technology to be easily replaced with newer technology. This is accomplished in the architecture of the SAP testbed by dividing it into multiple blocks that each has a different purpose and clearly defining the interfaces between each of these blocks. While this may not always facilitate the replacement of components in the testbed, it at least helps the designer have a better idea of how the different components interact and what is needed when replacing them. For example, if the BLE SoC were to be replaced but there is a desire to keep the other components in the system the same, then it is necessary to find an SoC that has a SPI interface, an ADC channel, and can operate at $2.6 \mathrm{~V}$.

\section{Conclusion}

BSNs address the weaknesses of traditional remote patient monitoring methods in healthcare and have emerged as an option to provide the opportunity for the continuous collection of high-quality data. However, the obstacles of user acceptance and battery life must be addressed before widespread adoption can occur. To address these obstacles, BSNs must be designed to be self-powered, wearable, and wireless. In addition, BSNs must have multimodal vigilant sensing so that they do not miss any critical events for their given application. While there are many applications for BSNs in healthcare, the one explored in this thesis is cardiac and activity monitoring. The SAP testbed is one such BSN that performs this kind of monitoring and it does so for the purpose of detecting critical cardiac and activity events that indicate the worsening of cardiovascular disease symptoms. This thesis presented the SAP testbed and discussed the design considerations that must be made when addressing the challenges involved in designing a BSN to be a self-powered wearable wireless multimodal vigilant sensing system. 
The major design challenges that were discussed include power management, energy harvesting optimization, system wearability optimization, and system flexibility and modularity optimization.

Power management is challenging because wireless communication and multimodal vigilant sensing consume large amounts of power, and when the energy harvester must be wearable, it can be difficult for it to generate enough power for the system to achieve self-powered operation long-term. To effectively manage system power consumption, ultra-low power components need to be used, energy storage devices and regulators need to be utilized, the wireless communication needs to be duty cycled, and an optimum sampling rate for the sensors that ensures both vigilant sensing and self-powered operation must be selected. Energy harvesting optimization is challenging because the size of some types of energy harvesters can impact both the amount of power available to the system and the system wearability, with larger harvesters generating more power but decreasing system wearability and vice versa. Energy harvesting can be optimized by placing the energy harvester in a location on the body that maximizes harvesting and by utilizing an efficient DC-DC converter. System wearability optimization is challenging because a self-powered BSN with multimodal sensing requires an energy harvester on the body and multiple sensors, which can make the system quite bulky. System wearability can be optimized by minimizing the size of the energy harvester, integrating most of the system components onto small printed circuit boards, and by integrating sensors into textiles. Finally, system flexibility and modularity optimization is challenging because there are many applications that must be considered during this process. This optimization can be accomplished by designing the BSN to be compatible with different energy harvesters, enabling it to have sensing modalities added or removed, and designing its architecture in such a way that enables older technology to be easily replaced with newer technology when it becomes available. The design of the SAP testbed addresses each of these challenges. 
The SAP testbed is a custom/COTS-based self-powered wearable sensor system designed to perform vigilant long-term cardiac and activity monitoring by continuously sensing and wirelessly streaming ECG and 3-axis motion data to a smartphone. It consumes only $370 \mu \mathrm{W}$ on average and is powered solely by indoor solar allowing it to maintain its ECG and motion monitoring at vigilant sampling rates of $25 \mathrm{~Hz}$ and $8.3 \mathrm{~Hz}$ respectively so that it does not miss any critical cardiac and activity events. The testbed was deployed on several subjects in an indoor laboratory environment with sufficient lighting and was able to operate continuously for long periods of time. It was also tested with fully-charged supercapacitors as the only power source in order to further characterize its performance. This characterization showed that given a large enough supercapacitor, the testbed can sustain vigilant self-powered operation for about 8.7 hours. This means that it can remain operational during extended periods of time with no ambient light. The testbed is also able to capture every QRS complex in the ECG signal for users with normal heart conditions as well as all small and large bodily movements from the motion data thus fulfilling its requirement of vigilant sensing. In addition, the small form factor of the SAP testbed, the flexible solar cells, and the integration of the different components onto the arm sleeve accomplish the goal of wearability for the testbed. While the SAP testbed fulfills its functional requirements, there are still efforts being made to improve it in various ways.

\section{Future Work}

One way in which the researchers in the ASSIST center are making efforts to improve the SAP testbed is by utilizing more custom components in order to further reduce its power consumption. Researchers at UVA are developing a custom SoC and researchers at UMich are developing a custom Bluetooth compliant radio to replace the DA14580 BLE SoC. With lower system power consumption, the solar cells would be able to sustain self-powered operation for longer periods of time under conditions with low ambient light. Also, the sampling rates of the sensors could be increased so that more features of the 
QRS complexes in the ECG signal could be detected. With more features detected, more critical events related to CVD symptoms could be monitored.

There are also efforts being made to increase the number of sensing modalities in the SAP testbed using custom sensors developed by researchers at FIU. This could improve the cardiac and activity monitoring of the testbed or even allow it to be used in other applications. Furthermore, researchers at NCSU are exploring ways to improve the integration of sensors into textiles to improve system wearability.

In addition to improvements to the functionality of the testbed, there are efforts being made to improve the technology readiness level of the current version of it so that the system can soon be deployed on CVD patients or patients with atrial fibrillation. One way that this is being addressed is by utilizing the OTP memory of the BLE SOC so that the user no longer has to worry about programming it, thereby improving the procedure that must be followed to make it operational. Once it is deployed on patients, the relationship between the level of patient activity and critical cardiac symptoms can be investigated as well as the compliance of patients when wearing a body-worn system. Also, a more accurate vigilant sampling rate for ECG monitoring that considers extreme heart conditions can be determined. 


\section{List of References}

[1] M. A. Hanson et al., "Body Area Sensor Networks: Challenges and Opportunities," Computer (Long. Beach. Calif)., vol. 42, no. 1, pp. 58-65, 2009.

[2] V. Weisfeld and T. A. Lustig, The Future of Home Health Care: Workshop Summary. 2015.

[3] J. H. M. Bergmann and A. H. McGregor, "Body-worn sensor design: What do patients and clinicians want?," Ann. Biomed. Eng., vol. 39, no. 9, pp. 2299-2312, 2011.

[4] B. H. Calhoun et al., "Body sensor networks: A holistic approach from silicon to users BT - CYBERPHYSICAL SYSTEMS,” Proc. IEEE, vol. 100, no. 1, pp. 91-106, 2012.

[5] D. Lloyd-Jones et al., "Executive summary: Heart disease and stroke statistics-2010 update: A report from the american heart association," Circulation, vol. 121, no. 7, 2010.

[6] G. Giamouzis, A. Kalogeropoulos, and V. Georgiopoulou, "Hospitalization Epidemic in Patients With Heart Failure : Risk Factors, Risk Prediction, Knowledge Gaps , and Future Directions," J. Card. Fail., vol. 17, no. 1, pp. 54-75, 2011.

[7] S. F. Jencks, M. V. Williams, and E. A. Coleman, "Rehospitalizations among Patients in the Medicare Fee-for-Service Program," N. Engl. J. Med., vol. 360, no. 14, pp. 1418-1428, 2009.

[8] C. Davis, M. Bender, T. Smith, and J. Broad, "Feasibility and Acute Care Utilization Outcomes of a Post-Acute Transitional Telemonitoring Program for Underserved Chronic Disease Patients," Telemed J E Heal. , vol. 21, no. 9, pp. 705-13, 2015.

[9] "Electrocardiography." [Online]. Available: https://en.wikipedia.org/wiki/Electrocardiography. [Accessed: 08-Aug-2017].

[10] ACLS Medical Training, "Guide to Understanding ECG Artifacts." [Online]. Available: https://www.aclsmedicaltraining.com/blog/guide-to-understanding-ecg-artifact/. [Accessed: 08Aug-2017].

[11] ASSIST, "Research Thrusts." [Online]. Available: https://assist.ncsu.edu/research/. [Accessed: 23May-2017].

[12] L. González-Villanueva, S. Cagnoni, and L. Ascari, "Design of a wearable sensing system for human motion monitoring in physical rehabilitation," Sensors (Switzerland), vol. 13, no. 6, pp. 77357755, 2013.

[13] Y. Han et al., "A self-powered insole for humanmotion recognition," Sensors (Switzerland), vol. 16, no. 9, pp. 1-12, 2016.

[14] J. Dieffenderfer et al., "Low Power Wearable Systems for Continuous Monitoring of Environment and Health for Chronic Respiratory Disease," IEEE J. Biomed. Heal. Informatics, vol. 20, no. 5, pp. $1-1,2016$.

[15] A. Pokhara and B. Mishra, "Design Methodology for an Energy Neutral Health Monitoring Wireless Sensor Node," 2016. 
[16] Alive Technologies, "Alive Bluetooth Heart \& Activity Monitor." [Online]. Available: http://www.alivetec.com/alive-bluetooth-heart-activity-monitor/. [Accessed: 15-Jun-2017].

[17] F. Miao, Y. Cheng, Y. He, Q. He, and Y. Li, "A wearable context-aware ECG monitoring system integrated with built-in kinematic sensors of the smartphone," Sensors (Switzerland), vol. 15, no. 5, pp. 11465-11484, 2015.

[18] A. Tobola et al., "Self-powered Multiparameter Health Sensor," IEEE J. Biomed. Heal. Informatics, vol. 14, no. 8, pp. 1-1, 2017.

[19] Analog Devices, "Micropower, 3-Axis, $\pm 2 \mathrm{~g} / \pm 4 \mathrm{~g} / \pm 8 \mathrm{~g}$ Digital Output MEMS Accelerometer." [Online]. Available: http://www.analog.com/media/en/technical-documentation/datasheets/ADXL362.pdf.

[20] Texas Instruments, "LPV521 NanoPower, 1.8-V, RRIO, CMOS Input, Operational Amplifier." [Online]. Available: http://www.ti.com/lit/ds/symlink/lpv521.pdf.

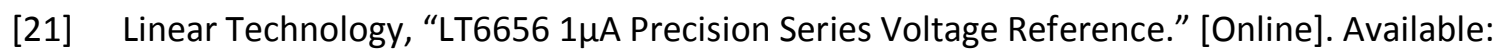
http://cds.linear.com/docs/en/datasheet/6656fc.pdf.

[22] PowerFilm Solar, "MP3-37." [Online]. Available:

http://www.powerfilmsolar.com/products/?mp337\&show=product\&productID=271535.

[23] N. O. A. Observatory, "Recommended Light Levels (Illuminance) for Outdoor and Indoor Venues," 2011. [Online]. Available:

https://www.noao.edu/education/QLTkit/ACTIVITY_Documents/Safety/LightLevels_outdoor+ind oor.pdf. [Accessed: 16-Aug-2017].

[24] Texas Instruments, "bq25504 Ultra Low-Power Boost Converter With Battery Management for Energy Harvester Applications." [Online]. Available:

http://www.ti.com/lit/ds/symlink/bq25504.pdf.

[25] Texas Instruments, "TPS783xx 500-nA IQ, 150-mA, Ultralow Quiescent Current Low-Dropout Linear Regulator." [Online]. Available: http://www.ti.com/lit/ds/symlink/tps783.pdf.

[26] Dialog Semiconductor, "DA14580 Bluetooth Low Energy 4.2 SoC." [Online]. Available: https://www.dialog-semiconductor.com/sites/default/files/da14580_ds_3v4.pdf.

[27] LinkLabs, "Bluetooth Vs. Bluetooth Low Energy: What's The Difference?" [Online]. Available: https://www.link-labs.com/blog/bluetooth-vs-bluetooth-low-energy. [Accessed: 12-Aug-2017].

[28] D. Scansen, "Thermoelectric Energy Harvesting." [Online]. Available: https://www.digikey.com/en/articles/techzone/2011/oct/thermoelectric-energy-harvesting. [Accessed: 13-Aug-2017].

[29] D. Fan, L. Lopez Ruiz, J. Gong, and J. Lach, "EHDC: An Energy Harvesting Modeling and Profiling Platform for Body Sensor Networks," IEEE J. Biomed. Heal. Informatics, vol. PP, no. 99, 2017.

[30] L. Buechley and M. Eisenberg, "Fabric PCBs, electronic sequins, and socket buttons: Techniques for e-textile craft," Pers. Ubiquitous Comput., vol. 13, no. 2, pp. 133-150, 2009. 